\title{
Formation of rhythmic sorted bed forms on the continental shelf: an idealised model
}

\author{
Tomas Van Oyen ${ }^{1} \dagger+$, Huib de Swart ${ }^{2}$ \\ and Paolo Blondeaux ${ }^{1}$ \\ ${ }^{1}$ Department of Civil, Environmental and Architectural Engineering, University of Genoa, \\ Via Montallegro 1, 16145 Genova, Italy \\ 2 Institute for Marine and Atmospheric research Utrecht, Utrecht University, IMAU, PO Box 80005, \\ NL-3508 TA Utrecht, The Netherlands
}

(Received 18 October 2010; revised 3 June 2011; accepted 13 July 2011)

An idealised model is presented to study the formation of sorted bed forms generated by a wind-driven along-shore current. The study employs a linear stability analysis to describe the time development of perturbations of both bottom composition and bed elevation, superimposed on a flat bed composed of a sediment mixture homogeneously distributed in space. The model considers both bed and suspended loads and takes into account the averaged influence of waves on the flow field and the transport of sediment. The results show that the positive coupling between waves, along-shore current and the erodible heterogeneous bed leads to the amplification of two modes, which exhibit distinct characteristics. A first mode is found to be dominant when moderate hydrodynamic conditions are considered and is primarily amplified by the convergence of sediment transport induced by the changes in the bed elevation. This mode has wavelengths of the order of hundred metres and has coarse (fine) sediments in its troughs (crests). By increasing the height of the waves and/or the strength of the steady current, the second mode can become dominant. This mode is characterised by shorter wavelengths and results from the interaction between the convergence of sediment transport related to changes in the bottom composition and that induced by perturbations of the bed elevation. These bed features can have an up-current or a down-current shift between the centre of the coarse-grained bands and the trough of the bottom wave. Typical growth times of the amplified features are of the order of hundreds of days and the migration rates, in the direction of the along-shore current, range between 0.1 and $10 \mathrm{~m}$ per day. A qualitative comparison of the model results with field observations indicates that the generation of two distinct modes provides a possible explanation for the broad range of characteristics of the natural bed features.

Key words: sediment transport

\section{Introduction}

Sorted bed forms (or ripple scour depressions) are intriguing bed features characterised by alternating bands of coarse and fine grains with wavelengths of

$†$ Email address for correspondence: tomas.vanoyen@ugent.be

\$ Present address: Department of Civil Engineering, Ghent University, Technology Park Campus Ardoyen, Building 904, B-9052 Ghent, Belgium. 
the order of hundred metres. Typical values of the mean grain size range between $0.1-0.4 \mathrm{~mm}$ and $0.6-2.0 \mathrm{~mm}$ in the fine and coarse regions, respectively. These bed forms are ubiquitously observed and exhibit a broad range of characteristics (e.g. Aubrey, Twichell \& Pfirman 1982; Cacchione, Grant \& Tate 1984; Goff et al. 2005; Ferrini \& Flood 2005; Bellec et al. 2010). Figure 1 shows a side scan sonar image of the inner shelf near Wrightsville Beach, North Carolina (USA), which reveals the presence of sorted bed forms in this area. Light shades indicate relatively coarse sand, whilst dark shades refer to fine sand.

Commonly, sorted bed forms are reported to be accompanied only by small variations of the bed elevation. In particular, the amplitude of the bathymetric undulations is observed to be smaller than or of the order of $1 \mathrm{~m}$, in mean water depths of $\approx 10 \mathrm{~m}$ (Murray \& Thieler 2004). Nevertheless, sorted bed forms with relatively larger amplitude also occur. For instance, Goff et al. (2005) describe bed features with heights of $3 \mathrm{~m}$, where the mean water depth is $\approx 12 \mathrm{~m}$.

Measurements performed along transects parallel to the shoreline indicate that the bands of coarse grains are typically located in the bathymetric lows, although a significant phase shift is often reported between the centre of the topographic low and the centre of the coarse patch. In general, the latter is observed on the down-current side of the trough (with respect to the direction of the dominant along-shore current; Schwab et al. 2000; Murray \& Thieler 2004). However, Ferrini \& Flood (2005) report, for the bed features along the south shore of Long Island, both down-current and up-current offsets. Moreover, observations at Martha's Vineyard Coastal Observatory, Massachusetts, reveal that the relative position of the coarse patch with respect to the bottom waviness can change proceeding along a sorted bed form from the shoreline to the offshore region (Goff et al. 2005).

The bed features often migrate, although their migration speed is found to differ significantly from site to site. Goff et al. (2005) report that the boundaries of the bed forms can migrate tens of metres in months, while the bed features observed in the German Bight, southeastern North Sea, appear essentially stable over a period of 26 years (Diesing et al. 2006).

In the past, sorted bed forms have been associated with locally intensified cross-shore flows related to coastal downwelling, taking place during storm events (Cacchione et al. 1984). More recent observations have shown that along-shore currents rather than cross-shore currents dominate the formation and maintenance of sorted bed forms (Thieler, Schwab \& Cacchione 1998; Thieler et al. 2001; Gutierrez, Voulgaris \& Thieler 2005). Motivated by these observations, Murray \& Thieler (2004) developed an exploratory model, which illustrates that sorted bed forms can originate as self-organising features due to the interaction of fractional sediment transport, bottom composition and turbulence. Conceptually, the model of Murray \& Thieler (2004) considers that the bottom roughness is larger at locations where the bed is coarser. This leads to locally more intense turbulence, which inhibits the settling of the finer sediment, thereby enhancing the coarse character of the bottom composition. Coco, Murray \& Green (2007a) extended the exploratory model of Murray \& Thieler (2004), replacing some idealised formulations with accepted and empirically based parametrisations. The results of Coco et al. (2007a) confirm the concept coined by Murray \& Thieler (2004), since with their model too, sorted bed forms are found to grow from the feedback between roughness-induced turbulence and fractional sediment transport. Moreover, using the model of Coco et al. $(2007 a, b)$ showed that less regular sorted bed forms, as reported by Ferrini \& Flood (2005), can also emerge from the 


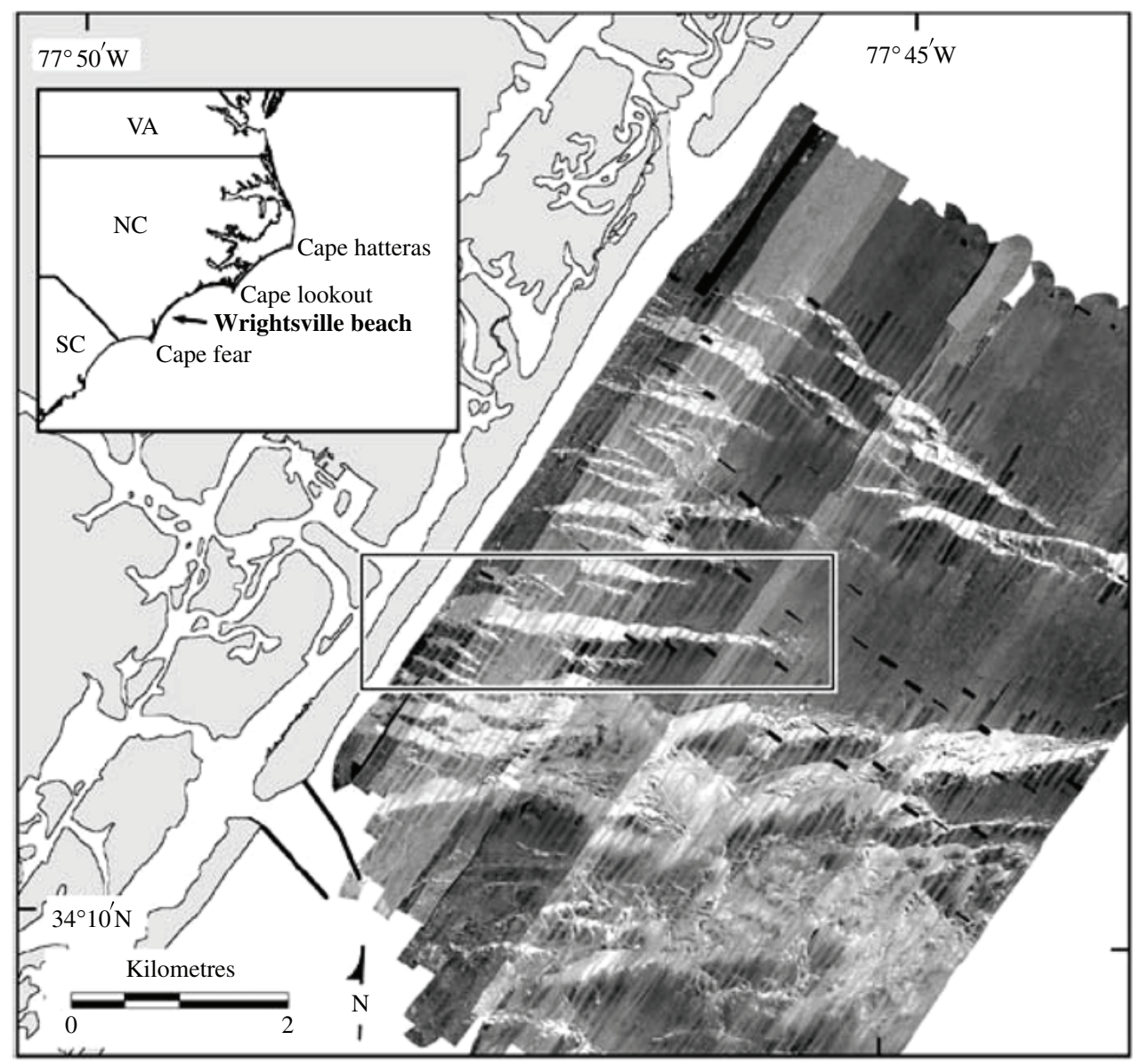

FIGURE 1. Side scan sonar map of the inner shelf off Wrightsville Beach, North Carolina (USA). The data reveal the presence of alternating stripes of coarse sand, resulting in a high back scatter (light), and fine sand (dark). Bathymetric data (not shown) reveal that these stripes are associated with (sorted) bed forms, where the coarse sand is found in the troughs and the fine sand at the crests. From Gutierrez et al. (2005), adapted from Thieler et al. (2001). Reproduced with permission of Elsevier.

feedback process described above, when complex hydrodynamic forcing scenarios are considered.

Although the models discussed above are quite successful, several aspects still remain unsolved. A primary question is related to the role of steady storm-driven along-shore currents in generating sorted bed forms. Indeed, with the models of Murray \& Thieler (2004) and Coco et al. (2007a), sorted bed forms characterised by a finite wavelength are only found by considering a symmetric reversing current. On the other hand, field observations of flow velocities at $1 \mathrm{~m}$ above the bed on the inner shelf of Wrightsville Beach show that storm-driven along-shore currents are dominant in one direction and control the sea bottom morphology (Gutierrez et al. 2005).

The aim of the present contribution is to investigate the conditions leading to the formation of sorted bed forms, focusing on the role of a steady wind-driven along- 
shore current. We follow the concept put forward by Murray \& Thieler (2004) and consider sorted bed forms to arise as self-organising features. However, a different approach is employed and the formation of sorted bed forms is analysed within the framework of a linear stability analysis. Thus, small-amplitude perturbations of bottom composition and bed elevation are superimposed on a flat bed with a spatially homogeneous distributed sediment, and a linear analysis of their time development is made.

The approach and methods presented here are similar to those employed by Colombini \& Parker (1995) and Seminara, Colombini \& Parker (1996) to investigate the formation of longitudinal streaks in wide erodible channels and bed load sheets in creeks, respectively. Colombini \& Parker (1995) showed that the correction to the flow field due to spatial variations of the roughness leads to the formation of longitudinal streaks for any value of the bottom shear stress above the threshold value for the initiation of sediment motion, while variations in bed elevation induce streak formation only when high values of the bottom shear stress are considered. Seminara et al. (1996) showed that perturbations of the bottom composition alone can lead to the formation of bed load sheets in creeks characterised by large Froude numbers.

On the inner shelf, sediment sorting patterns along shoreface-connected ridges have been studied by Walgreen, de Swart \& Calvete (2003), de Swart et al. (2008) and Vis-Star, de Swart \& Calvete (2009). These bed forms differ from sorted bed forms both in length and orientation. Indeed, shoreface-connected ridges are elongated bed forms with crest-to-crest distance of the order of a few kilometres and amplitudes of a few metres. Moreover, these bed forms are connected to the shoreface and extend seaward towards the outer shelf, forming an angle of $20^{\circ}-30^{\circ}$ with respect to the shoreline.

A brief description of the main idea at the basis of the analysis is given in Van Oyen, de Swart \& Blondeaux (2010). The present paper builds upon this work and is aimed at providing a detailed description of the idealised model and the model output. Moreover, an interpretation of the results is presented and the theoretical predictions are compared with field data collected at different locations to explore the ability of the model to describe the characteristics of the sorted bed forms for different values of the input parameters. In the following section, the formulation of the hydrodynamic and morphodynamic problems is described. Next, we consider the time development and interaction of small periodic perturbations of the bottom composition and the bed elevation. In $\S 4$, the results obtained are presented and theoretically interpreted. A discussion of the results is provided in $\S 5$ and concluding remarks are given in the last section.

\section{The model}

An idealised model is formulated to describe the linear interaction of a steady wind-driven along-shore current with small perturbations of the bottom composition and bed elevation. As is explained below, the idealisation concerns simplifications made in describing the geometry (e.g. no lateral boundaries), the flow field (e.g. no tidal currents) and the sediment characteristics (e.g. only two different grain sizes). Regarding the processes that are included in the model, state-of-the-art formulations are adopted.

Based on field observations, a recent tentative summary performed by Coco et al. (2007b) indicates that the bed features can be classified into three types according to their shape: patchy, V-shaped and linear. The focus of the present study is on the 


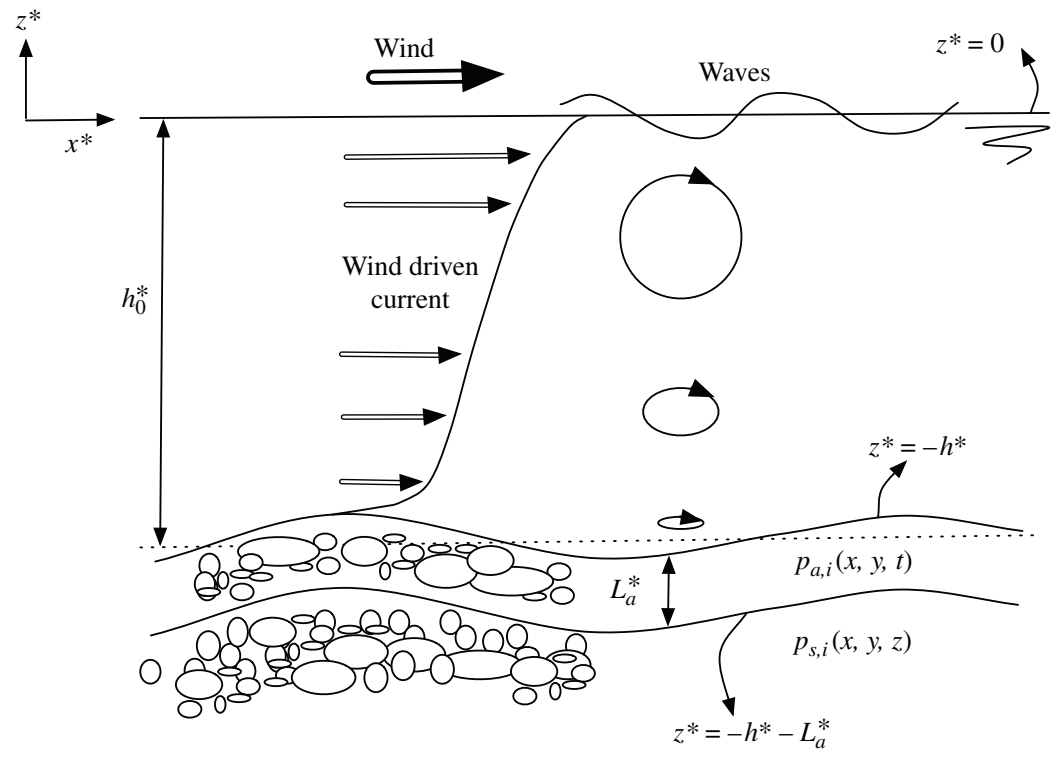

FIGURE 2. Side view of the water column in the along-shore direction illustrating the hydrodynamic and morphodynamic problem.

formation of sorted bed forms of the linear type. Since these bed forms are observed to have crests that are approximately perpendicular to the shoreline and are elongated up to a few kilometres from the shoreface into the sea, a simplified model geometry is used. Following earlier models of sorted bed forms, the effects of the slope of the inner shelf are neglected and a horizontal bottom is considered. Although this assumption could appear crude, it allows us to focus on the appearance of sorted bed forms, filtering out the generation of shoreface-connected sand ridges which, in general, requires a sloping bed. Furthermore, the hydrodynamic and sedimentologic interaction between the shoreface and the inner shelf can be assumed to be of minor importance, such that the model geometry can be considered uniform and unbounded in the horizontal directions. An orthogonal coordinate system is adopted with $x^{*}$ - and $z^{*}$-axes pointing in the along-shore and vertical directions, respectively. The vertical axis points upwards and $z^{*}=0$ corresponds to the still water level. The $x^{*}$-axis is taken aligned with the direction of the along-shore current. Hereinafter a star denotes a dimensional quantity. Figure 2 illustrates the model geometry and provides a sketch of the hydrodynamic and morphodynamic problem discussed below.

\subsection{Hydrodynamic problem}

The hydrodynamic problem consists of the flow field which is driven by a steady wind, blowing in the along-shore direction, and is affected by the presence of sea waves. In the following, all quantities are wave-averaged quantities, since our interest is not in intra-wave phenomena.

We assume the steady along-shore current to be turbulent and we neglect molecular viscosity. A Boussinesq-type approach is employed to evaluate the Reynolds stresses and a scalar kinematic eddy viscosity $v_{T}^{*}$ is introduced. The equations of motion are written in dimensionless form using the mean water depth $h_{0}^{*}$ as the length scale. The fluid velocity components $u^{*}$ and $w^{*}$ in the horizontal and vertical directions, 
respectively, are made dimensionless using the depth-averaged along-shore current velocity $U_{0}^{*}$. Furthermore, the dimensionless variable $v_{T}=v_{T}^{*} / v_{T 0}^{*}$ is introduced, in which $v_{T 0}^{*}$ and $v_{T}(z)$ denote the scale and vertical profile of the eddy viscosity, respectively. Finally, for later convenience, we introduce the dynamic pressure $p^{*}$, which is related to the pressure $P^{*}$ by $p^{*}=P^{*}+\rho^{*} g^{*} z^{*}$, and we make it dimensionless using $\rho^{*} U_{0}^{* 2}\left(\rho^{*}\right.$ denotes the sea water density and $g^{*}$ represents the gravitational acceleration).

In dimensionless form, the equations governing the currents read

$$
\begin{gathered}
\frac{\partial u}{\partial x}+\frac{\partial w}{\partial z}=0 \\
u \frac{\partial u}{\partial x}+w \frac{\partial u}{\partial z}=-\frac{\partial p}{\partial x}+\frac{1}{R_{t}}\left\{\frac{\partial}{\partial x}\left(2 v_{T} \frac{\partial u}{\partial x}\right)+\frac{\partial}{\partial z}\left[v_{T}\left(\frac{\partial u}{\partial z}+\frac{\partial w}{\partial x}\right)\right]\right\} \\
u \frac{\partial w}{\partial x}+w \frac{\partial w}{\partial z}=-\frac{\partial p}{\partial z}+\frac{1}{R_{t}}\left\{\frac{\partial}{\partial x}\left[v_{T}\left(\frac{\partial w}{\partial x}+\frac{\partial u}{\partial z}\right)\right]+\frac{\partial}{\partial z}\left(2 v_{T} \frac{\partial w}{\partial z}\right)\right\} .
\end{gathered}
$$

Here, it is assumed that the flow adapts instantaneously to changes in the bed profile, exploiting the significant difference between the characteristic time scales of the flow and the bed evolution. In (2.1)-(2.3), the Reynolds number $R_{t}=h_{0}^{*} U_{0}^{*} / \nu_{T 0}^{*}$ appears.

Considering typical values of $U_{0}^{*}$ and $h_{0}^{*}\left(\sim 0.3 \mathrm{~m} \mathrm{~s}^{-1}\right.$ and $10 \mathrm{~m}$, respectively), the flow field turns out to be characterised by a small Froude number $F_{r}=U_{0}^{*} / \sqrt{g^{*} h_{0}^{*}}$. Hence, the rigid-lid approximation can be applied and the effects of the free surface elevation on the local depth can be neglected. This approximation has no direct consequences for (2.1)-(2.2), but it has implications for the formulations of the eddy viscosity $v_{T}$ and for the boundary conditions, to be discussed hereafter.

The influence of sea waves on the steady current is accounted for by adopting a suitable eddy viscosity profile. As pointed out by Fredsøe \& Deigaard (1992, p. 77), far from the surf region where wave breaking is absent, the turbulence structure is significantly affected by sea waves only in the region closest to the bed; while in the upper region of the fluid column, the turbulent mixing of momentum is dominated by the current. In this study, the eddy viscosity model proposed by Christoffersen \& Jonsson (1985, Model II) is adopted. Hence, $v_{T}$ increases linearly with the distance from the bed within the wave bottom boundary layer $\delta_{w}\left(=\delta_{w}^{*} / h_{0}^{*}\right)$ and has the profile suggested by Dean (1974) in the rest of the fluid column. The latter resembles closely the standard parabolic profile, except that it achieves a finite non-zero value at the free surface. As illustrated by Colombini \& Stocchino (2005), the use of such a profile is reasonable when pure wind-driven flows are considered. Therefore, the vertical profile of the dimensionless kinematic eddy viscosity reads

$$
v_{T}= \begin{cases}(z+h) u_{f w} & \text { if } z \leqslant-h+\delta_{w}, \\ \frac{\hat{\xi}(1-\hat{\xi})}{1+2 \mathscr{A} \hat{\xi}^{2}+3 \mathscr{B} \hat{\xi}^{3}} h u_{f c} & \text { if } z>-h+\delta_{w},\end{cases}
$$

where $\hat{\xi}=(z+h) / h, \mathscr{A}=1.84$ and $\mathscr{B}=-1.56$. The scale of the kinematic eddy viscosity is equal to $v_{T 0}^{*}=\kappa h_{0}^{*} u_{f c 0}^{*}$. Here, $\kappa$ represents the von Kármán constant and $u_{f c 0}^{*}$ denotes the scale of the local friction velocity exerted by the current, considering the flow field over a flat bed with a spatially homogeneous distributed bottom composition. Furthermore, $h=h^{*} / h_{0}^{*}$ represents the dimensionless local depth and $u_{f w}=u_{f w}^{*} / u_{f c 0}^{*}$ is a dimensionless variable which denotes the maximum of the friction velocity during a wave period due to the combined action of the current and the near-bed wave orbital 
velocity. Furthermore, the dimensionless current-related friction velocity is denoted by $u_{f c}=u_{f c}^{*} / u_{f c 0}^{*}$. The profile of $v_{T}$ described by (2.4) is discontinuous at $z=-h+\delta_{w}$. However, for numerical convenience, $v_{T}(z)$ is made continuous by introducing a thin layer where the two profiles are matched.

The current-related friction velocity $u_{f c}^{*}=\sqrt{\tau_{c}^{*} / \rho^{*}}$ is computed by evaluating the current-related bed shear stress $\tau_{c}^{*}$, which reads

$$
\tau_{c}^{*}=\rho^{*} \nu_{T}^{*}\left[2 n_{x} n_{z}\left(\frac{\partial u^{*}}{\partial x^{*}}-\frac{\partial w^{*}}{\partial z^{*}}\right)+\left(n_{z}^{2}-n_{x}^{2}\right)\left(\frac{\partial u^{*}}{\partial z^{*}}+\frac{\partial w^{*}}{\partial x^{*}}\right)\right]_{z^{*}=-h^{*}+h_{b}^{*}} .
$$

Here, $\left(n_{x}, n_{z}\right)$ denote the components of the unit vector normal to the bed described by $z^{*}=-h^{*}$. The bed shear stress is evaluated at a distance $h_{b}^{*}$ above the bed. The meaning of, and an expression for $h_{b}^{*}$ will be given further on.

The direction of wave propagation with respect to the shoreline is highly variable in space and time, but it is commonly found to be approximately perpendicular to the shore. Therefore, and also to keep the analysis relatively simple, the waves are assumed to propagate in the direction orthogonal to the along-shore current. In this case, $u_{f w}^{*}$ is given by

$$
u_{f w}^{*}=\sqrt{\frac{\tau_{m}^{*}}{\rho^{*}}} \text { with } \tau_{m}^{*}=\sqrt{\left(\tau_{c}^{*}\right)^{2}+\left(\hat{\tau}_{w}^{*}\right)^{2}} \text { and } \hat{\tau}_{w}^{*}=\frac{1}{2} \rho^{*} f_{w}\left(\hat{u}_{w b}^{*}\right)^{2} .
$$

Here, $f_{w}$ is the wave friction factor and $\hat{u}_{w b}^{*}$ denotes the amplitude of the near-bed wave orbital velocity. The latter is evaluated using the linear wave theory, assuming the height and wavelength of the sea waves to be known. To evaluate the wave friction factor, the formulation proposed by Soulsby (1997) is used:

$$
f_{w}=1.39\left(\frac{A_{e}}{z_{r} / \chi}\right)^{-0.52},
$$

where $A_{e}=A_{e}^{*} / h_{0}^{*}, A_{e}^{*}=\hat{u}_{w b}^{*} / \omega_{w}^{*}$ is the wave excursion length, $\omega_{w}^{*}$ is the angular wave frequency and $z_{r}=z_{r}^{*} / h_{0}^{*}$ is the dimensionless bottom roughness. Finally, the dimensionless thickness of the wave bottom boundary layer is determined following Christoffersen \& Jonsson (1985):

$$
\delta_{w}=\frac{0.367 \kappa u_{f w}^{*}}{h_{0}^{*} \omega_{w}^{*}} .
$$

To evaluate the bottom roughness, sea ripples induced by the action of waves are supposed to be present at the bed. The empirical formula proposed by Soulsby \& Whitehouse (2005) is used to compute the dimensionless bottom roughness due to wave ripples,

$$
z_{r}=0.15\left[1-\exp \left\{-\left(5000 / \Delta_{\text {sed }}\right)^{3.5}\right\}\right] \lambda,
$$

and the dimensionless wavelength $\lambda$ of the sea ripples $\left(\lambda=\lambda^{*} / h_{0}^{*}\right)$,

$$
\lambda=A_{e}\left\{1+1.87 \times 10^{-3} \Delta_{\text {sed }}\left[1-\exp \left(-2.0 \times 10^{-4} \Delta_{\text {sed }}\right)^{1.5}\right]\right\}^{-1},
$$

where

$$
\Delta_{s e d}=\frac{h_{0}^{*}}{d_{g m, 0}^{*}} \frac{A_{e}}{d_{g m}} .
$$


The variable $d_{g m}^{*}$ denotes the local geometric mean grain size of the sediment mixture and is made dimensionless with $d_{g m, 0}^{*}$, which is the geometric mean grain size of the initial bottom composition, i.e. considering a flat bed without horizontal variations in the bottom composition.

The hydrodynamic problem is closed by imposing appropriate boundary conditions. At the bottom, the fluid velocity is forced to vanish at a distance $z_{r} / \chi$ from the bed level $(\chi=29.8)$. Because of the rigid-lid approximation introduced previously, the variations in the free surface level on the local depth are neglected. Therefore, the bottom profile is described by $z=-h$ ( $h$ being the local depth) and the dynamic boundary conditions at the free surface are imposed at $z=0$ :

$$
\frac{v_{T}}{R_{t}}\left(\frac{\partial w}{\partial x}+\frac{\partial u}{\partial z}\right)=\frac{\tau_{w i n d}^{*}}{\rho^{*}\left(U_{0}^{*}\right)^{2}}, \quad-p+\frac{2}{R_{t}} v_{T} \frac{\partial w}{\partial z}=0 \quad \text { at } z=0 .
$$

Here, $\tau_{\text {wind }}^{*}$ is the dimensional wind shear stress in the along-shore direction which drives the steady current. Finally, applying the kinematic boundary condition, we impose $w=0$ at $z=0$.

\subsection{Morphodynamic problem}

\subsubsection{Sediment mass balance}

A heterogeneous cohesionless sediment mixture composed of $N$ distinct grain size classes is considered. Each grain size class $n$ is associated with a grain size diameter $d_{n}^{*}$ and has a probability $p_{n}$ of occurrence in the mixture. Although the values of $p_{n}$ can vary in space and time, they are subject to the constraint

$$
\sum_{n=1}^{N} p_{n}=1 .
$$

For most sediments, the probabilities $p_{n}$ follow approximately a log-normal distribution and can be assumed to be adequately described by the first and second moments of the distribution (see Lanzoni \& Tubino 1999, and references therein). Therefore, we introduce the $\phi$-scale and define

$$
\phi_{n}=-\log _{2}\left(\frac{d_{n}^{*}}{d_{r e f}^{*}}\right),
$$

in which $d_{r e f}^{*}$ is equal to $1 \mathrm{~mm}$. Furthermore, the sediment mixture is characterised by its geometric mean grain size

$$
d_{g m}^{*}=d_{r e f}^{*} 2^{-\phi_{m}} \quad \text { with } \phi_{m}=\sum_{n=1}^{N} p_{n} \phi_{n}
$$

and by its standard deviation $\sigma$, where

$$
\sigma^{2}=\sum_{n=1}^{N}\left(\phi_{n}-\phi_{m}\right)^{2} p_{n} .
$$

Considering a sediment mixture, the conservation of sediment mass can be written in terms of the availability for transport of each grain size class. This involves the evaluation of the probability $p_{n}$ throughout the entire bed, while observations indicate that only the top layer of the bed takes part in the local instantaneous sediment transport process. This top layer (or active layer) scales with the mean grain size when 
the bed is flat, and is of the order of the ripple height when small-scale bed forms are present. Based on these considerations, Hirano (1971) introduced the active layer model. This model assumes that $p_{s, n}$, which is the probability of occurrence of a grain size class $n$ in the substrate (the entire bed underneath the active layer), is independent of time but it can have an arbitrary vertical profile. Inside the (active) top layer, the sediment is well mixed and characterised by a probability of occurrence $p_{a, n}$ which can depend on $t$, but does not depend on $z$.

Applying this model, the sediment continuity equation for each grain size class reads

$$
-p_{c, n} \frac{\partial}{\partial t^{*}}\left(h^{*}+L_{a}^{*}\right)+\frac{\partial}{\partial t^{*}}\left(p_{a, n} L_{a}^{*}\right)=-\frac{1}{\left(1-p_{o r}\right)} \frac{\partial \mathscr{Q}_{n}^{*}}{\partial x^{*}},
$$

where $p_{\text {or }}$ denotes the sediment porosity. In (2.17), $p_{c, n}$ represents the volume fraction of grain size class $n$ that is exchanged between the substrate and the active layer. The active layer thickness is given by $L_{a}^{*}$ and is taken to be equal to $z_{r}^{*}$, since ripples are present. Furthermore, $\mathscr{Q}_{n}^{*}$ represents the volumetric sediment transport rate per unit width of the class $n$ and is equal to $p_{a, n} q_{n}^{*}\left(q_{n}^{*}\right.$ is the sediment transport rate per unit volume fraction).

Introducing the dimensionless variables

$$
L_{a}=\frac{L_{a}^{*}}{h_{0}^{*}}, \quad t=\frac{t^{*} U_{0}^{*}}{h_{0}^{*}}, \quad \mathscr{Q}_{n}=\frac{\mathscr{Q}_{n}^{*}}{\sqrt{(s-1) g^{*}\left(d_{g m, 0}^{*}\right)^{3}}},
$$

the sediment continuity equation for each grain size class reads

$$
-p_{c, n} \frac{\partial}{\partial t}\left(h+L_{a}\right)+\frac{\partial}{\partial t}\left(p_{a, n} L_{a}\right)=-\beta \frac{\partial \mathscr{Q}_{n}}{\partial x} .
$$

The dimensionless parameter $\beta$, which appears in (2.19), is given by

$$
\beta=\frac{d_{g m, 0}^{*}}{h_{0}^{*}\left(1-p_{o r}\right) \sqrt{\psi_{m}}},
$$

while the sediment mobility number $\psi_{m}$ is defined as

$$
\psi_{m}=\frac{\left(U_{0}^{*}\right)^{2}}{(s-1) g^{*} d_{g m, 0}^{*}} .
$$

Finally, $s$ is the ratio between the density of the sediment and that of the water.

Summation over all grain size classes leads to an equation for the time development of the bed level,

$$
\frac{\partial h}{\partial t}=\beta \sum_{n=1}^{N} \frac{\partial \mathscr{Q}_{n}}{\partial x} .
$$

To close the morphodynamic problem, a description of the transport of sediment due to the combined action of currents and waves is required. We consider $q_{n}$ as the sum of the bedload transport, a correction on that transport due to the presence of bed slopes, and suspended load transport: $q_{n}=q_{b, n}+q_{s l, n}^{b}+q_{s, n}$. In this study, the relationships suggested by Kleinhans \& van Rijn (2002), following earlier work by van Rijn (1991), are adopted to quantify these transport contributions. 


\subsubsection{Bed load transport}

The bed load transport formulation reads

$$
q_{b, n}= \begin{cases}\sqrt{\frac{d_{n}^{*}}{d_{g m, 0}^{*}}} \frac{0.25 d_{g m}}{R_{p, g m}^{0.2}} \sqrt{\mu_{c} \theta_{c, n}}\left(\frac{\theta_{c w, n}-\theta_{c r, n}^{b}}{\theta_{c r, g m}^{b}}\right)^{3 / 2} & \text { if } \theta_{c w, n}>\theta_{c r, n}^{b}, \\ 0 & \text { if } \theta_{c w, n} \leqslant \theta_{c r, n}^{b},\end{cases}
$$

where $R_{p, g m}$ denotes the sediment Reynolds number of the bottom material,

$$
R_{p, g m}=\frac{\sqrt{d_{g m}^{*}{ }^{3}(s-1) g^{*}}}{\nu^{*}},
$$

$v^{*}$ is the kinematic sea water viscosity, and $\tau_{w}^{*}$ is the wave-related mean bed shear stress given by

$$
\tau_{w}^{*}=\frac{1}{4} \rho^{*} f_{w}\left(\hat{u}_{w b}^{*}\right)^{2} .
$$

In (2.23), $\theta_{c, n}$ and $\theta_{c w, n}$ represent the Shields parameters related to the current only and due to the combined action of currents and waves, respectively. They are defined by

$$
\theta_{c, n}=\frac{\tau_{c}^{*}}{\rho^{*}(s-1) g^{*} d_{n}^{*}} \quad \text { and } \quad \theta_{c w, n}=\frac{\mu_{c} \tau_{c}^{*}+\mu_{w, n} \tau_{w}^{*}}{\rho^{*}(s-1) g^{*} d_{n}^{*}}
$$

where $\mu_{w, n}$ is a wave efficiency factor,

$$
\mu_{w, n}=\frac{0.6}{R_{p, n}^{2 / 3}} \quad \text { with } R_{p, n}=\frac{\sqrt{d_{n}^{* 3}(s-1) g^{*}}}{v^{*}},
$$

and the parameter $\mu_{c}$ denotes an efficiency factor, such that the shear stress due to the current is reduced with the part that is related to the form drag. Specifically,

$$
\mu_{c}=\left(\frac{\tilde{C}}{\hat{C}}\right)^{2}
$$

where

$$
\tilde{C}=5.75 \log _{10}\left(\frac{12 h^{*}}{z_{r}^{*}}\right), \quad \hat{C}=5.75 \log _{10}\left(\frac{12 h^{*}}{3 d_{g m}^{*} 2^{\sigma}}\right)
$$

are the conductance coefficients related to the roughness and to the characteristics of the sediment mixture, respectively (van Rijn 1991).

The final parameter that appears in (2.23) is the critical Shields parameter $\theta_{c r, n}^{b}$ for each grain size class $n$. For this, we adopt the formulation of Ashida \& Michiue (1972), which accounts for the hiding of small grains between the large grains. 
Thus, $\theta_{c r, n}^{b}=\mathscr{H}_{n} \theta_{c r, g m}^{b}$ with

$$
\mathscr{H}_{n}= \begin{cases}{\left[\frac{\log _{10}(19)}{\log _{10}\left(19 \frac{d_{n}^{*}}{d_{g m}^{*}}\right)}\right]^{2}} & \text { when } \frac{d_{n}^{*}}{d_{g m}^{*}}>0.4, \\ 0.843 \frac{d_{g m}^{*}}{d_{n}^{*}} & \text { when } \frac{d_{n}^{*}}{d_{g m}^{*}} \leqslant 0.4 .\end{cases}
$$

Furthermore, $\theta_{c r, g m}^{b}$ denotes the critical Shields parameter of the mean grain size and is evaluated following Brownlie (1981):

$$
\theta_{c r, g m}^{b}=0.22 R_{p, g m}^{-0.6}+0.06 \exp \left(-17.77 R_{p, g m}^{-0.6}\right) .
$$

We include hiding, because previous studies on grain-sorting phenomena (Seminara et al. 1996; Walgreen et al. 2003; Vis-Star et al. 2009; Van Oyen \& Blondeaux 2009) show that it is an important factor controlling the transport of a sediment mixture and the grain size distribution.

The sediment transport due to bedslope effects is quantified following Kovacs \& Parker (1994),

$$
q_{s l, n}^{b}=\frac{\theta_{c r, n}^{b}}{\hat{\mu}} \frac{d q_{b, n}}{d \theta_{c, n}} \frac{\partial h}{\partial x},
$$

with $\hat{\mu}$ a dynamic friction factor, which is presently assumed equal to 0.57 .

\subsubsection{Suspended load transport}

To calculate the suspended sediment transport rate, it is first necessary to compute the sediment volume concentration $c_{n}$ of each grain size class $n$, which is governed by an advection-diffusion equation

$$
\frac{\partial}{\partial x}\left(u c_{n}\right)+\frac{\partial}{\partial z}\left(w c_{n}-\frac{w_{s, n} c_{n}}{\sqrt{\psi_{n}}}\right)=\frac{1}{R_{t}}\left\{\frac{\partial}{\partial x}\left(\mathscr{K}_{T} \frac{\partial c_{n}}{\partial x}\right)+\frac{\partial}{\partial z}\left(\mathscr{K}_{T} \frac{\partial c_{n}}{\partial z}\right)\right\} .
$$

In (2.33), $w_{s, n}\left(=w_{s, n}^{*} / \sqrt{(s-1) g^{*} d_{n}^{*}}\right)$ denotes the dimensionless fall velocity of the grain size fraction $n$ and is calculated following Parker (1978),

$$
\log _{10}\left(w_{s, n}\right)=-1.181+0.966 \pi_{p, n}-0.1804 \pi_{p, n}^{2}+0.003746 \pi_{p, n}^{3}+0.0008782 \pi_{p, n}^{4},
$$

where $\pi_{p, n}=\log _{10} R_{p, n}$. Furthermore, $\mathscr{K}_{T}=\mathscr{K}_{T}^{*} / v_{T 0}^{*}$ represents the sediment eddy diffusivity. Considering the discussion in Fredsøe \& Deigaard (1992, pp. 227-234), in the following, the profile of $\mathscr{K}_{T}$ is assumed equal to that of the kinematic eddy viscosity $v_{T}$.

In order to solve the advection-diffusion equation (2.33), appropriate boundary conditions are required. At the free surface, the sediment flux in the normal direction vanishes,

$$
\frac{w_{s, n}}{\sqrt{\psi_{n}}} c_{n}+\frac{\mathscr{K}_{T}}{R_{t}} \frac{\partial c_{n}}{\partial z}=0 \quad \text { at } z=0
$$


while at the reference level $z=-h+a$, we prescribe

$$
-\frac{\mathscr{K}_{T}}{R_{t}} \frac{\partial c_{n}}{\partial z}=\frac{w_{s, n}}{\sqrt{\psi_{n}}} c_{r e f, n} .
$$

Following van Rijn $(1984,1991)$, the reference level is considered to be $1 \%$ of the local depth above the seabed $(a=0.01 h)$, and the expression for $c_{r e f, n}$ reads

$$
c_{r e f, n}=\frac{d_{g m, 0}^{*}}{h_{0}^{*}} \frac{0.015 d_{g m}}{a R_{p, g m}^{0.2}}\left(\frac{\theta_{c w, n}-\theta_{c r, n}^{b}}{\theta_{c r, g m}^{b}}\right)^{3 / 2} .
$$

The reference concentration $c_{r e f, n}$ is set to zero when the Shields parameter $\theta_{n}$ is smaller than the critical Shields parameter for suspension $\theta_{c r, n}^{s}$, which is given by

$$
\theta_{c r, n}^{s}= \begin{cases}\left(\frac{4 w_{s, n}}{R_{p, n}^{2 / 3}}\right)^{2} & \text { if } R_{p, n} \leqslant 31.6227, \\ \left(0.4 w_{s, n}\right)^{2} & \text { if } R_{p, n}>31.6227,\end{cases}
$$

as is proposed by van Rijn (1993).

Once the vertical concentration profile is obtained, the suspended sediment transport rate is evaluated, neglecting the horizontal diffusion and integrating the product of the velocity and the sediment concentration over the water depth,

$$
q_{s, n}=\sqrt{\psi_{m}} \frac{h_{0}^{*}}{d_{\text {mean }, 0}^{*}} \int_{-h+a}^{0} u(z) c_{n}(z) \mathrm{d} z .
$$

Finally, note that, following the analysis of Colombini (2004), we evaluate the current-related friction velocity, the near-bed wave orbital velocity and $u_{f w}$ at the top of the bed load layer $h_{b}^{*}$. The value of $h_{b}^{*}$ is computed as the maximum of $h_{b, n}^{*}$ :

$$
h_{b, n}^{*}=z_{r}^{*}\left[1+1.3\left(\frac{\theta_{c w, n}-\theta_{c r, n}^{b}}{\theta_{c r, g m}^{b}}\right)^{0.55}\right] .
$$

\section{Linear stability analysis and solutions}

The aim of the present analysis is to investigate the linear time development of small periodic perturbations of both bottom composition and bed elevation, superimposed on our basic state: a flat bed with a spatially homogeneous distributed sediment composition. A linear analysis is performed such that the spatial components of the perturbations of the local depth and bottom composition evolve independently of each other and the problem is solved for the generic spatial component

$$
\begin{gathered}
p_{a, n}=p_{a 0, n}+\epsilon p_{a 1, n}(t) \exp \left[\mathrm{i} \alpha_{x} x\right]+\text { c.c. }+O\left(\epsilon^{2}\right), \\
h=1-\epsilon h_{1}(t) \exp \left[\mathrm{i} \alpha_{x} x\right]+\text { c.c. }+O\left(\epsilon^{2}\right) .
\end{gathered}
$$

In (3.2), $\epsilon p_{a 1, n}(t)$ and $\epsilon h_{1}(t)$ are the amplitudes of the generic component of the perturbation of the bottom composition and the local depth, respectively. The variable $\alpha_{x}$ represents the wavenumber of the perturbation, and $\epsilon \ll 1$.

The small value of $\epsilon$ allows us to expand the hydrodynamic variables in terms of $\epsilon$ :

$$
\begin{aligned}
\left(u, w, p, u_{f c}, u_{f w}, v_{T}\right)= & \left(u_{0}, w_{0}, p_{0}, u_{f c 0}, u_{f w 0}, v_{T 0}\right) \\
& +\epsilon\left(\hat{u}_{1}, \hat{w}_{1}, \hat{p}_{1}, \hat{u}_{f c 1}, \hat{u}_{f w 1}, \hat{v}_{T 1}\right) \exp \left[i \alpha_{x} x\right]+\text { c.c. }+O\left(\epsilon^{2}\right) .
\end{aligned}
$$


Furthermore, the changes in the bottom composition lead to perturbations in the mean grain size and in the standard deviation of the sediment mixture, which can be written as

$$
\left(\phi_{m}, d_{g m}, \sigma\right)=\left(\phi_{m 0}, 1, \sigma_{0}\right)+\epsilon\left(\phi_{m 1}, d_{g m 1}, \sigma_{1}\right) \exp \left[\mathrm{i} \alpha_{x} x\right]+\text { c.c. }+O\left(\epsilon^{2}\right) .
$$

The relation between the perturbation of the geometric mean grain size and $\phi_{m 1}$ reads

$$
d_{g m 1}=-\ln 2 \phi_{m 1} .
$$

Although the problem is formulated for an arbitrary number of distinct grain size classes and no problem exists to deal with three or more grain size classes, in the following, to simplify the algebra and the discussion of the results, the sediment mixture is assumed to consist of two grain sizes only. Then, considering the constraint on the volume fractions $\left(p_{1}+p_{2}=1\right)$, it follows that $p_{a 1,1}+p_{a 1,2}=0$ such that $\phi_{m 1}$ is given by

$$
\phi_{m 1}(t)=\left(\phi_{1}-\phi_{2}\right) p_{a 1,1}(t),
$$

while the contribution at order $\epsilon$ to the standard deviation reads

$$
\sigma_{1}=\frac{1}{2 \sigma_{0}}\left(\phi_{1}^{2}-\phi_{2}^{2}-2 \phi_{m 0}\left(\phi_{1}-\phi_{2}\right)\right) p_{a 1,1} \text {. }
$$

Furthermore, expanding the empirical relations for $f_{w}, \lambda$ and $z_{r}$ in terms of $\epsilon$,

$$
\left(f_{w}, \lambda, z_{r}\right)=\left(f_{w 0}, \lambda_{0}, z_{r 0}\right)+\epsilon\left(\hat{f}_{w 1}, \hat{\lambda}_{1}, \hat{z}_{r 1}\right) \exp \left[\mathrm{i} \alpha_{x} x\right]+\text { c.c. }+O\left(\epsilon^{2}\right),
$$

the first-order contributions $\hat{f}_{w 1}, \hat{\lambda}_{1}$ and $\hat{z}_{r 1}$ turn out to be proportional to $p_{a 1,1}$, such that we can write

$$
\left(\hat{f}_{w 1}, \hat{\lambda}_{1}, \hat{z}_{r 1}\right)=\left(f_{w 1}, \lambda_{1}, z_{r 1}\right) p_{a 1,1} .
$$

Equation (3.9) illustrates that the variations in the bottom composition affect the ripple geometry (height and wavelength), such that changes in the bottom composition are equivalent to variations in the bottom roughness. In particular, the variables $z_{r 1}$ and $\lambda_{1}$ turn out to be real and positive, which means that over the coarser-grained regions, the ripple height and wavelength are larger than in the fine-grained regions.

Finally, we also expand the sediment transport rates for each grain size class:

$$
\left(q_{b, n}, q_{s, n}, q_{s l, n}^{b}\right)=\left(q_{b 0, n}, q_{s 0, n}, q_{s l 0, n}^{b}\right)+\epsilon\left(\hat{q}_{b 1, n}, \hat{q}_{s 1, n}, \hat{q}_{s l 1, n}^{b}\right) \exp \left[\mathrm{i} \alpha_{x} x\right]+\text { c.c. }+O\left(\epsilon^{2}\right) \text {. }
$$

Substitution of (3.2)-(3.10) into the equations previously described leads to hydrodynamic and morphodynamic problems to orders $\epsilon^{0}$ and $\epsilon^{1}$.

\subsection{Basic state}

To the leading order of approximation, the hydrodynamic problem consists of the evaluation of a steady wind-driven along-shore current over a flat bed with a homogeneous bottom composition. Combining the hydrodynamic boundary conditions with momentum and continuity (2.1)-(2.3) yields

$$
w_{0}=0, \quad p_{0}=0, \quad \frac{v_{T 0}(z)}{R_{t}} \frac{\mathrm{d} u_{0}}{\mathrm{~d} z}=\frac{\tau_{\text {wind }}^{*}}{\rho^{*}\left(U_{0}^{*}\right)^{2}} .
$$


By integrating the horizontal momentum balance over depth, it follows that the bottom shear stress $\tau_{c 0}^{*}=\rho^{*}\left(u_{f c 0}^{*}\right)^{2}$ equals the wind stress, hence

$$
u_{f c 0}^{*}=\sqrt{\frac{\tau_{w i n d}^{*}}{\rho^{*}}} .
$$

Next, the vertical profile of $u_{0}$ is evaluated by numerically integrating (3.11) with a finite difference approach, i.e. a second-order Runge-Kutta method. Note that the numerical integration of (3.11) requires the knowledge of both $U_{0}^{*}$ and $\tau_{\text {wind }}^{*}$, which are coupled. Therefore, an iterative procedure is adopted. In particular, we fix the depth-averaged mean current velocity and iterate on $\tau_{\text {wind }}^{*}$ until the correct value of $U_{0}^{*}$ is obtained.

\subsection{Perturbed state}

To order $\epsilon$, a linear set of equations governing the perturbed flow field is obtained:

$$
\begin{gathered}
\frac{\partial \hat{w}_{1}}{\partial z}=-\mathrm{i} \alpha_{x} \hat{u}_{1} \\
\mathrm{i} \alpha_{x} u_{0} \hat{u}_{1}+\hat{w}_{1} \frac{\mathrm{d} u_{0}}{\mathrm{~d} z}=-\mathrm{i} \alpha_{x} \hat{p}_{1}+\frac{1}{R_{t}}\left\{-2 v_{T 0} \alpha_{x}^{2} \hat{u}_{1}+\frac{\partial}{\partial z}\left(v_{T 0} \frac{\partial \hat{u}_{1}}{\partial z}\right)\right. \\
\left.+\frac{\partial}{\partial z}\left(\hat{v}_{T 1} \frac{\mathrm{d} u_{0}}{\mathrm{~d} z}\right)+\mathrm{i} \alpha_{x} \hat{w}_{1} \frac{\mathrm{d} v_{T 0}}{\mathrm{~d} z}+\mathrm{i} \alpha_{x} v_{T 0} \frac{\partial \hat{w}_{1}}{\partial z}\right\} \\
-\mathrm{i} \alpha_{x} u_{0} \hat{w}_{1}=-\frac{\partial \hat{p}_{1}}{\partial z}+\frac{1}{R_{t}}\left\{-v_{T 0} \alpha_{x}^{2} \hat{w}_{1}\right. \\
\left.+v_{T 0} \mathrm{i} \alpha_{x} \frac{\partial \hat{u}_{1}}{\partial z}+\mathrm{i} \alpha_{x} \hat{v}_{T 1} \frac{\mathrm{d} u_{0}}{\mathrm{~d} z}+2 \frac{\partial}{\partial z}\left(v_{T 0} \frac{\partial \hat{w}_{1}}{\partial z}\right)\right\}
\end{gathered}
$$

The linearised boundary conditions at the free surface $z=0$ are given by

$$
\hat{w}_{1}=0, \quad v_{T 0} \frac{\partial \hat{u}_{1}}{\partial z}+\hat{v}_{T 1} \frac{\mathrm{d} u_{0}}{\mathrm{~d} z}=0, \quad 2 \frac{v_{T 0}}{R_{t}} \frac{\partial \hat{w}_{1}}{\partial z}=\hat{p}_{1},
$$

while the bottom boundary conditions read

$$
\hat{u}_{1}=-\left(\frac{\mathrm{d} u_{0}}{\mathrm{~d} z}\right) \frac{z_{r 1}}{\chi} p_{a 1,1}-\left(\frac{\mathrm{d} u_{0}}{\mathrm{~d} z}\right) h_{1}, \quad \hat{w}_{1}=0 \quad \text { at } z=-1+z_{r 0} / \chi .
$$

The first bottom boundary condition explicitly shows that the perturbed flow is linearly related to the perturbations of the bed elevation and of the bottom composition, denoted by subscripts $h$ and $p$, respectively. Hence, it is possible to decompose the solution into

$$
\begin{aligned}
\left(\hat{u}_{1}, \hat{w}_{1}, \hat{p}_{1}, \hat{u}_{f c 1}, \hat{u}_{f w 1}, \hat{v}_{T 1}\right)= & \left(u_{1, h}, w_{1, h}, p_{1, h}, u_{f c 1, h}, u_{f w 1, h}, v_{T 1, h}\right) h_{1}(t) \\
& +\left(u_{1, p}, w_{1, p}, p_{1, p}, u_{f c 1, p}, u_{f w 1, p}, v_{T 1, p}\right) p_{a 1,1}(t) .
\end{aligned}
$$

Substituting (3.18) into (3.13)-(3.16), two independent problems are obtained, one for the terms proportional to $p_{a 1,1}(t)$ and the other for the terms proportional to $h_{1}(t)$. 
When the flow over a periodic perturbation of the bottom composition is considered, the bottom boundary conditions read

$$
u_{1, p}=-\left(\frac{\mathrm{d} u_{0}}{\mathrm{~d} z}\right) \frac{z_{r 1}}{\chi}, \quad w_{1, p}=0 \quad \text { at } z=-1+z_{r 0} / \chi .
$$

These conditions illustrate that the flow field due to the perturbation in the bottom composition is entirely induced by the bottom roughness changes, which, in turn, follow from the variations in $p_{a 1,1}$ (see (3.9)). On the other hand, the boundary conditions at the bottom for the flow induced by periodic variations in the bed elevation require

$$
u_{1, h}=-\left(\frac{\mathrm{d} u_{0}}{\mathrm{~d} z}\right), \quad w_{1, h}=0 \quad \text { at } z=-1+z_{r 0} / \chi .
$$

Both problems are solved by numerically integrating the ordinary differential equations obtained by substitution of (3.18) into (3.13)-(3.16), in the $z$-direction, from the bottom up to the free surface. A second-order Runge-Kutta method is used, and the solution which satisfies both the boundary conditions at the free surface and at the bottom is obtained using a shooting procedure.

The functions $v_{T 1, h}$ and $v_{T 1, p}$ introduced in (3.18) depend on $u_{f c 1, h}$ and $u_{f c 1, p}$, respectively. In turn, $u_{f c 1, h}$ and $u_{f c 1, p}$ are related to the order $\epsilon$ contributions to the eddy viscosity:

$$
\begin{aligned}
& u_{f c 1, p}=\frac{1}{2 R_{t}}\left(\frac{U_{0}^{*}}{u_{f c 0}^{*}}\right)^{2}\left\{v_{T 1, p} \frac{\mathrm{d} u_{0}}{\mathrm{~d} z}+v_{T 0}\left[\frac{\partial u_{1, p}}{\partial z}+\mathrm{i} \alpha_{x} w_{1, p}\right]\right\}, \\
& u_{f c 1, h}=\frac{1}{2 R_{t}}\left(\frac{U_{0}^{*}}{u_{f c 0}^{*}}\right)^{2}\left\{v_{T 1, h} \frac{\mathrm{d} u_{0}}{\mathrm{~d} z}+v_{T 0}\left[\frac{\partial u_{1, h}}{\partial z}+\mathrm{i} \alpha_{x} w_{1, h}\right]\right\},
\end{aligned}
$$

evaluated at $z=-1+h_{b}$. Therefore, the hydrodynamic problems at order $\epsilon$ are also solved using an iterative procedure.

Once the flow field at order $\epsilon$ is known, the perturbations of the Shields parameters are evaluated,

$$
\left(\theta_{c, n}, \theta_{c w, n}\right)=\left(\theta_{c 0, n}, \theta_{c w 0, n}\right)+\left(\hat{\theta}_{c 1, n}, \hat{\theta}_{c w 1, n}\right) \exp \left[\mathrm{i} \alpha_{x} x\right]+\text { c.c. }+O\left(\epsilon^{2}\right) .
$$

Like the flow field, the first-order corrections of $\theta_{c, n}$ and $\theta_{c w, n}$ can also be split into two parts,

$$
\left(\hat{\theta}_{c 1, n}, \hat{\theta}_{c w 1, n}\right)=\left(\theta_{c 1, h, n}, \theta_{c w 1, h, n}\right) h_{1}+\left(\theta_{c 1, p, n}, \theta_{c w 1, p, n}\right) p_{a 1,1} .
$$

Next, taking into account (3.10), it follows that

$$
\begin{gathered}
\left(\hat{q}_{b 1, n}, \hat{q}_{s 1, n}\right)=\left(q_{b 1, h, n}, q_{s 1, h, n}\right) h_{1}+\left(q_{b 1, p, n}, q_{s 1, p, n}\right) p_{a 1,1}, \\
\hat{q}_{s 11, n}^{b}=q_{s l 1, h, n}^{b} h_{1} .
\end{gathered}
$$

Physically, (3.25) and (3.26) show that the spatial variations of both the bed elevation and the bottom composition lead to changes in the sediment transport rate. These changes are induced 'directly' and 'indirectly'. In other words, the sediment transport rate alters since the flow field near the bed is affected by changes in the local depth and by changes in the bottom roughness (composition). Indirectly, the mixing of momentum is also affected by the variations in $h_{1}$ and $p_{a 1,1}$ (see (3.18)). Hence, since the present model considers the profile of the diffusion of sediment concentration to 
be the same as that of the mixing of momentum, the sediment diffusivity also differs over the coarser-grained regions in comparison with the finer-grained regions, and in the troughs with respect to the crests.

Next, we analyse the sediment continuity equations at order $\epsilon$,

$$
p_{a 0, n} \frac{\mathrm{d} h_{1}}{\mathrm{~d} t}+L_{a 0} \frac{\mathrm{d} p_{a 1, n}}{\mathrm{~d} t}=-\beta \mathrm{i} \alpha_{x}\left(q_{1, p, n} p_{a 1,1}+q_{0, n} p_{a 1, n}+q_{1, h, n} h_{1}\right),
$$

in which the bed composition of the basic state is assumed to be homogeneous, hence $p_{c 0, n}=p_{a 0, n}$. Moreover,

$$
\begin{gathered}
q_{1, p, n}=p_{a 0, n} q_{b 1, p, n}+p_{a 0, n} q_{s 1, p, n}, \quad q_{1, h, n}=p_{a 0, n}\left(q_{b 1, h, n}+q_{s 1, h, n}+q_{s l 1, h, n}^{b}\right), \\
q_{0, n}=q_{b 0, n}+q_{s 0, n} .
\end{gathered}
$$

Summation over both grain sizes leads to an equation which describes the time development of the changes in the bed elevation:

$$
\frac{\mathrm{d} h_{1}}{\mathrm{~d} t}=-\beta \mathrm{i} \alpha_{x}\left[S_{p} p_{a 1,1}(t)+S_{h} h_{1}(t)\right] .
$$

The expressions of $S_{p}$ and $S_{h}$ are given in the Appendix. Back-substitution of (3.30) into (3.27) yields a system of two equations for $p_{a 1,1}(t)$ and $h_{1}(t)$ :

$$
\begin{gathered}
\frac{\mathrm{d} p_{a 1,1}}{\mathrm{~d} t}=-\mathrm{i} \alpha_{x} \frac{\beta}{L_{a 0}}\left\{\left(q_{1, p, 1}+q_{0,1}-p_{a 0,1} S_{p}\right) p_{a 1,1}+\left(q_{1, h, 1}-p_{a 0,1} S_{h}\right) h_{1}\right\}, \\
\frac{\mathrm{d} h_{1}}{\mathrm{~d} t}=-\mathrm{i} \alpha_{x} \beta\left(S_{p} p_{a 1,1}+S_{h} h_{1}\right) .
\end{gathered}
$$

The solution to (3.32) is obtained by writing

$$
p_{a 1,1}(t)=\tilde{p} \mathrm{e}^{\gamma t}, \quad h_{1}(t)=\tilde{h} \mathrm{e}^{\gamma t},
$$

such that the system of equations can be written as a linear eigenvalue problem:

$$
\left[\begin{array}{cc}
\mathscr{L}_{p 1} & \mathscr{L}_{h 1} \\
\mathscr{L}_{p 2} & \mathscr{L}_{h 2}
\end{array}\right]\left[\begin{array}{l}
\tilde{p} \\
\tilde{h}
\end{array}\right]=\gamma\left[\begin{array}{l}
\tilde{p} \\
\tilde{h}
\end{array}\right]
$$

In the Appendix the expressions of $\mathscr{L}_{p 1}, \mathscr{L}_{p 2}, \mathscr{L}_{h 1}$ and $\mathscr{L}_{h 2}$ are provided.

The complex values of $\gamma$ are obtained considering the non-trivial solutions of the eigenvalue problem described by (3.34). The two eigenvalues $\gamma_{1}$ and $\gamma_{2}$ are found from the characteristic equation and read

$$
\gamma_{1}=\frac{\mathscr{G}+\sqrt{\mathscr{G}^{2}-4 \mathscr{D}}}{2} \text { and } \gamma_{2}=\frac{\mathscr{G}-\sqrt{\mathscr{G}^{2}-4 \mathscr{D}}}{2} .
$$

The expressions of $\mathscr{G}$ and $\mathscr{D}$ are specified in the Appendix.

From (3.33), it follows that the real part of the eigenvalues controls the growth of the perturbations in the bottom composition and the bed elevation, while the imaginary part is the frequency. Moreover, each eigenvalue $\gamma_{1}$ and $\gamma_{2}$ corresponds to an eigenvector $\boldsymbol{v}_{1}$ and $\boldsymbol{v}_{2}$, respectively:

$$
\boldsymbol{v}_{1}=\left(\tilde{p}_{1}, \tilde{h}_{1}\right) \quad \text { and } \quad \boldsymbol{v}_{2}=\left(\tilde{p}_{2}, \tilde{h}_{2}\right) .
$$


From the eigenvalue problem (3.34), the ratio between $\tilde{h}$ and $\tilde{p}$ can be determined and it is given by

$$
\frac{\tilde{h}_{1}}{\tilde{p}_{1}}=\frac{\mathscr{L}_{p 2}}{\gamma_{1}-\mathscr{L}_{h 2}}=\mathscr{R}_{1} \quad \text { and } \quad \frac{\tilde{h}_{2}}{\tilde{p}_{2}}=\frac{\mathscr{L}_{p 2}}{\gamma_{2}-\mathscr{L}_{h 2}}=\mathscr{R}_{2} .
$$

Hence, for each eigenvector, the relative amplitude of the bed level perturbation with respect to the amplitude of the perturbation of the bottom composition can be evaluated by computing $\left|\mathscr{R}_{1}\right|$ and $\left|\mathscr{R}_{2}\right|$. Furthermore, the ratio $\mathscr{R}_{k}$ between $\tilde{h}_{k}$ and $\tilde{p}_{k}$ $(k=1,2)$ also describes the phase difference $\Psi_{k, \text { ang }}$ between their maxima, i.e.

$$
\Psi_{k, \text { ang }}=\arctan \left(\frac{\mathscr{R}_{k, r}}{\mathscr{R}_{k, i}}\right)-\frac{\pi}{2}+\left\{\begin{array}{ll}
0 & \text { if }-\left(\mathscr{R}_{k, i}\right) \geqslant 0 \\
\pi & \text { if }-\left(\mathscr{R}_{k, i}\right)<0
\end{array} \quad(k=1,2),\right.
$$

where the under scripts $r$ and $i$ denote the real and imaginary parts of a complex quantity, respectively. Assuming the coarse grains of the bimodal grain size mixture to be represented by the grain size class 1, the region of coarse grains is found upstream (downstream) of the trough with respect to the direction of the along-shore current when $\Psi_{\text {ang }}$ is between 0 and $\pi(\pi$ and $2 \pi)$.

\section{Model applications to field cases, interpretation of the modes and sensitivity experiments}

In this section, we analyse the initial free response of the morphodynamic system to the imposed perturbations, and investigate whether these perturbations amplify in time and lead to the generation of sorted bed forms. As pointed out by Murray et al. (2005) and Coco et al. (2007a), model results can depend significantly on the parametrisations adopted (even if well-established relationships are used) when the level of detail of the model formulation is high. Therefore, instead of providing a full quantitative description of the results as functions of the input parameters, first we investigate the model outputs, taking into account input values representative of three locations at which sorted bed forms are observed. The theoretical findings are discussed in detail in the second part of this section, after which the free response of the morphodynamic system is determined considering different hydrodynamic conditions.

\subsection{The field cases}

First, the bed features observed at Wrightsville Beach, North Carolina, are considered (Thieler et al. 2001; Murray \& Thieler 2004; Gutierrez et al. 2005; see also figure 1). At this site, sorted bed forms, which are observed from depths of 3-4 m to depths of $\sim 15 \mathrm{~m}$, have crests which are almost orthogonal to the coastline and have a wavelength ranging between 40 and $200 \mathrm{~m}$. The bands of coarser grains are observed in the bathymetric lows, with the centre of the coarse patch shifted downstream to the centre of the trough (with respect to the direction of the main along-shore current). Inside the regions of coarse grains, the mean grain size is approximately $1.1 \mathrm{~mm}$, while outside these regions the mean grain size is around $0.2 \mathrm{~mm}$.

Gutierrez et al. (2005) describe measurements of the near-bed current at two sites on the inner shelf, near Wrightsville Beach. At both locations, the current velocity and pressure data were measured at $1 \mathrm{~m}$ above the bed during a 45-day period in the late winter/early spring of 1996. During the entire measurement campaign, Gutierrez et al. (2005) report six storm events during which the sediment is assumed to be set into 


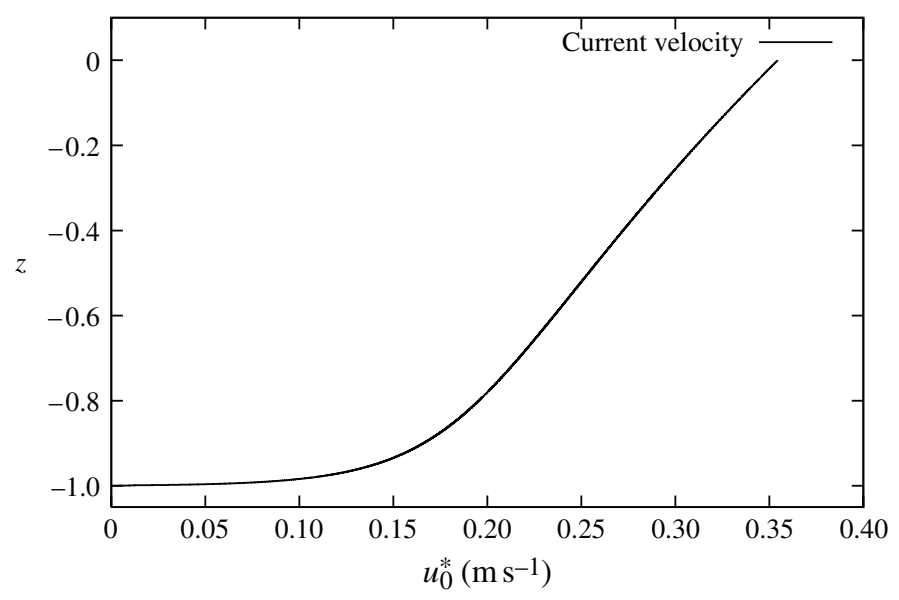

FIGURE 3. Vertical profile of the wind-driven current velocity for depth-averaged current speed $U_{0}^{*}=0.25 \mathrm{~m} \mathrm{~s}^{-1}$, depth $h_{0}^{*}=10 \mathrm{~m}$, wave height $H_{w}^{*}=1.0 \mathrm{~m}$ and wavelength $\lambda_{w}^{*}=$ $60 \mathrm{~m}$. The bottom roughness $z_{r 0}^{*}$ is approximately $5.5 \mathrm{~cm}$ and the grain size of the coarse and fine fraction is $1.15 \mathrm{~mm}$ and $0.2 \mathrm{~mm}$, respectively.

motion. During such events, the amplitude of the current velocity at one metre above the bed ranges between $0.1 \mathrm{~m} \mathrm{~s}^{-1}$ and $0.2 \mathrm{~m} \mathrm{~s}^{-1}$ while the near-bed wave orbital velocity is between $0.25 \mathrm{~m} \mathrm{~s}^{-1}$ and $0.4 \mathrm{~m} \mathrm{~s}^{-1}$. Finally, wave periods range from 6 to $7 \mathrm{~s}$.

Therefore, as model inputs, we consider a mean water depth of $10 \mathrm{~m}$ and a bimodal mixture characterised by $d_{1}^{*}$ and $d_{2}^{*}$ equal to $1.15 \mathrm{~mm}$ and $0.2 \mathrm{~mm}$, respectively. Hereinafter, in the basic state, the bottom composition is assumed to be composed of an equal amount of fine and coarse grains, such that $p_{a 0,1}=p_{a 0,2}=0.5$. To model the hydrodynamic conditions, a depth-averaged along-shore current velocity $U_{0}^{*}=0.25 \mathrm{~m} \mathrm{~s}^{-1}$ is considered and the sea waves are supposed to be characterised by a waveheight $H_{w}^{*}=1 \mathrm{~m}$ and a wavelength $\lambda_{w}^{*}=60 \mathrm{~m}$, respectively. These input values lead to a near-bed wave orbital velocity of $0.35 \mathrm{~m} \mathrm{~s}^{-1}$ and a wave period of $7 \mathrm{~s}$. Furthermore, as illustrated in figure 3, a current velocity of $0.17 \mathrm{~m} \mathrm{~s}^{-1}$ is obtained at $1 \mathrm{~m}$ above the bed. To obtain a value of $U_{0}^{*}$ equal to $0.25 \mathrm{~m} \mathrm{~s}^{-1}$, the applied wind shear stress at the free surface turns out to be equal to $0.2 \mathrm{~N} \mathrm{~m}^{-2}$. Such a value, considering the friction factor proposed by Van Dorn (1953), corresponds to a wind speed of $9.4 \mathrm{~m} \mathrm{~s}^{-1}$ at $10 \mathrm{~m}$ above the free surface. This value agrees well with measurements of the wind speed near Wrightsville Beach during the storm events (Gutierrez et al. 2005).

In figure 4 , the maximum $\gamma_{\max , r}$ of the real parts of the two eigenvalues $\left(\gamma_{\max , r}=\max \left[\gamma_{1, r}, \gamma_{2, r}\right]\right)$ is plotted versus the dimensionless wavenumber $\alpha_{x}$. The growth rate is positive for values of $\alpha_{x}$ between 0 and 1.53 such that, in this range of wavenumbers, the initial perturbations are amplified in time. For larger values of $\alpha_{x}, \gamma_{\max , r}$ vanishes, which corresponds to marginally stable perturbations. As will be discussed more thoroughly later on, the latter result follows from the vanishing value of the real part of one of the two eigenvalues for all wavenumbers.

Within the range of amplified wavenumbers, $\gamma_{\max , r}$ attains a maximum when the dimensionless wavenumber is equal to 0.77 . The perturbation with this wavenumber $\alpha_{x, \max }$ is the fastest-growing mode. Thus, starting from random perturbations (all wavenumbers present), it is this mode that will dominate the solution after some 


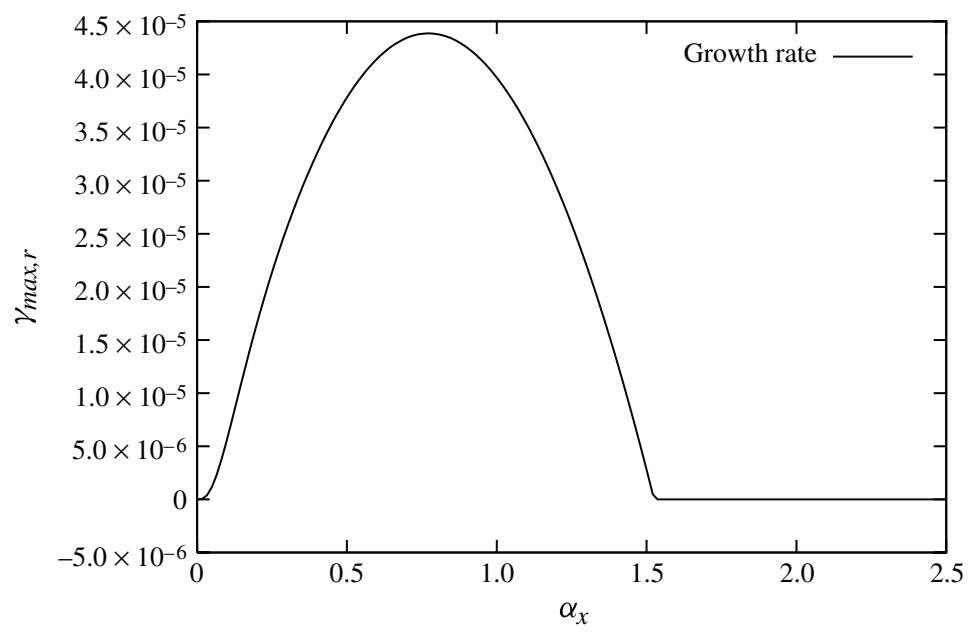

FIGURE 4. Maximum growth rate $\gamma_{\max , r}$ plotted versus the dimensionless wavenumber $\alpha_{x}$. Parameter values are representative of the conditions at Wrightsville Beach.

time. The value of the most amplified wavenumber $\alpha_{x, \max }$ corresponds to a dimensional wavelength $\lambda_{\max }^{*}$ of $82 \mathrm{~m}$, which is comparable with the wavelength of the observed bed features.

To analyse the relative position of the centre of the coarse patch and the trough of the bottom waviness, the value of $\Psi_{\text {ang }}$ is evaluated. For the most amplified mode, $\Psi_{\text {ang }}$ is equal to $\pi$, which means that the coarse grains are found in the bathymetric lows.

Finally, we determine the migration speed $c_{m i g}^{*}$ and the dimensional $e$-folding time scale $T_{\gamma}^{*}$ of the fastest-growing mode, which are given by

$$
c_{\text {mig }}^{*}=-\frac{\gamma_{\max , i}}{\alpha_{\max }} U_{0}^{*}, \quad T_{\gamma}^{*}=\frac{h_{0}^{*}}{U_{0}^{*} \gamma_{\max , r}} .
$$

Here, $\gamma_{\max , i}$ is the imaginary part of the eigenvalue with the largest real part. Taking into account that the storm events only occur during a time fraction of approximately 0.05 , the bed features are found to migrate $1.0 \mathrm{~m}$ per day in the downstream direction and $T_{\gamma}^{*}$ turns out to be $\sim 210$ days. The obtained values of $c_{m i g}^{*}$ and $T_{\gamma}^{*}$ agree with those found by Coco et al. (2007a), where the bed features attain a relative equilibrium after $\sim 40$ days and migrate approximately $5 \mathrm{~m}$ in one day (G. Coco, personal communication).

Next, a comparison of the model findings with field observations of sorted bed forms at Martha's Vineyard Coastal Observatory, Massachusetts, is performed (Goff et al. 2005). At this site, the bed features are observed in depths from 8 to $18 \mathrm{~m}$. They emerge shore-normal from the shoreface into the inner shelf and have wavelengths ranging from 100 to $500 \mathrm{~m}$. The bottom sediment at this site is bimodal and is composed of a fine fraction with a grain size approximately equal to $0.25 \mathrm{~mm}$ and a coarse fraction with grain sizes between $0.5 \mathrm{~mm}$ and $2.0 \mathrm{~mm}$. Furthermore, the boundaries of the bands of coarse grains are observed to migrate about tens of metres per year.

The bed features are reported to be asymmetric with respect to the shore-normal direction. The patches of coarse grains are characterised by one well-defined edge and one that is ragged, and the centre of the band of coarse grains is shifted with 


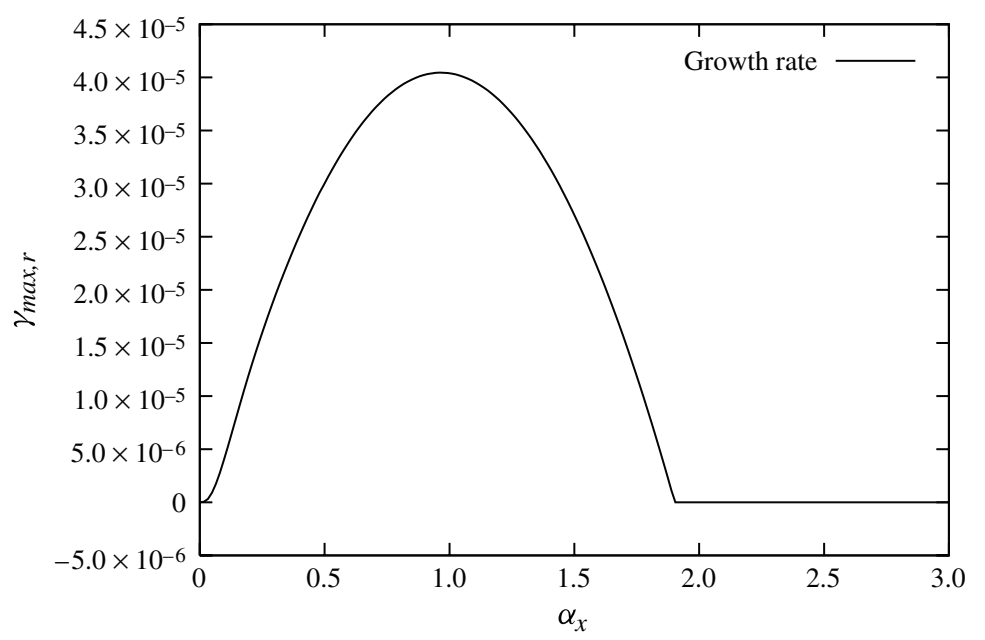

FIGURE 5. Maximum growth rate $\gamma_{\max , r}$ plotted versus the dimensionless wavenumber $\alpha_{x}$. Parameter values are representative of Martha's Vineyard Coastal Observatory, Massachusetts.

respect to the bathymetric low. Remarkably, within the survey area, the asymmetry is not the same for all the bed features, e.g. it changes from deep water towards shallow water. The bed features also exhibit a broad spectrum of bathymetric reliefs, i.e. the amplitude of the bottom waviness is of the order of $10 \mathrm{~cm}$ in the western part and it goes up to $3 \mathrm{~m}$ in the eastern region (Goff et al. 2005).

Model runs are performed with $h_{0}^{*}=14 \mathrm{~m}$ and a grain size mixture characterised by $d_{1}^{*}=0.75 \mathrm{~mm}$ and $d_{2}^{*}=0.25 \mathrm{~mm}$. Since the hydrodynamic conditions, to the authors' present knowledge, have not yet been analysed in detail, we follow Coco et al. (2007b) and suppose the wave conditions to be moderate: $H_{w}^{*}=1.5 \mathrm{~m}, \lambda_{w}^{*}=75 \mathrm{~m}$. Finally, the depth-averaged current velocity is considered to be $0.3 \mathrm{~m} \mathrm{~s}^{-1}$.

Figure 5 shows the value of $\gamma_{\max , r}$ plotted versus $\alpha_{x}$, using the input values representative of the conditions at Martha's Vineyard. Similar behaviour to that of figure 4 is obtained. The growth rate $\gamma_{\max , r}$ is positive for wavenumbers ranging between 0 and 1.9, after which the growth rate becomes zero. The most amplified mode is found for $\alpha_{x}$ equal to 0.96, such that the fastest-growing bed features have a wavelength of $92 \mathrm{~m}$. The $e$-folding time scale turns out to be $\sim 280$ days and $c_{m i g}^{*}$ is approximately $0.8 \mathrm{~m}$ per day. Furthermore, $\Psi_{\text {ang }}$ equals $\pi$ for the fastest-growing mode. Hence, the centre of the coarse grain fraction is entirely out of phase with the undulation of the bottom elevation.

Lastly, we compare the model results with data of the sorted bed forms observed by Aubrey et al. (1982) off Nauset Inlet, Cape Cod, Massachusetts. They have a wavelength of the order of hundreds of metres, with a 1-3 m relief when the depth is between 8 and $18 \mathrm{~m}$. More shoreward, the bed forms have a much smaller channel size and are related with more gentle changes of the bed elevation. The bottom composition in the troughs of the bathymetric undulations is composed of coarse grains, while the adjacent bed contains fine grains. In the region shoreward of the $8 \mathrm{~m}$ contour, the mean grain size in the coarse patch is $0.68 \mathrm{~mm}$ and in the surrounding area the mean grain size is $0.35 \mathrm{~mm}$ (Coco et al. 2007b). Aubrey et al. (1982) report that the area is subject to a moderate wave climate and describe that current velocities measured at $0.6 \mathrm{~m}$ above the bed (in depths of $10 \mathrm{~m}$ ) are $\sim 0.2 \mathrm{~m} \mathrm{~s}^{-1}$. 
In our model, we consider a mean water depth of $7 \mathrm{~m}$ and a depth-averaged velocity of $0.25 \mathrm{~m} \mathrm{~s}^{-1}$. The bottom composition is assumed to consist of coarse grains with a diameter equal to $0.65 \mathrm{~mm}$ and fine grains with a diameter equal to $0.35 \mathrm{~mm}$. Finally, waves are considered with a height and a wavelength equal to $1.5 \mathrm{~m}$ and $65 \mathrm{~m}$, respectively.

In figure $6(a)$, the maximum growth rate is plotted versus $\alpha_{x}$ for the conditions off Nauset Inlet. Different behaviour with respect to the previous two cases is found. Indeed, in this case the real parts of both eigenvalues are characterised by non-vanishing values. In figure $6(b)$, the values of $\gamma_{1, r}, \gamma_{2, r}$ and $\gamma_{\max , r}$ are plotted, concentrating on the behaviour of the growth rate related to the second eigenvalue. The value of $\gamma_{2, r}$ is positive for small wavenumbers up to bed forms characterised by a wavenumber equal to $\sim 0.6$. Next, for large values of $\alpha_{x}, \gamma_{2, r}$ attains a negative value. Moreover, the value of $\gamma_{2, r}$ has a maximum for $\alpha_{x}$ equal to 0.3 such that preferred bed features with a wavelength of approximately $145 \mathrm{~m}$ are predicted by the analysis for the second mode.

Nevertheless, figure 6 shows that the value of $\gamma_{1, r}$ is always larger than that of $\gamma_{2, r}$ when the growth rate related to the first eigenvalue is positive. Hence, the fastest-growing mode is related to the maximum value of $\gamma_{1, r}$ and is attained for bed forms with a wavelength equal to $115 \mathrm{~m}$. Furthermore, the value of $\Psi_{\text {ang }}$ is slightly larger than $\pi\left(\Psi_{\text {ang }}=1.005 \pi\right)$ such that the centre of the coarse patch is predicted in the bathymetric low slightly shifted in the down-current direction. The dimensional $e$-folding time scale is 670 days and the migration speed is equal to $0.5 \mathrm{~m}$ per day.

Hence, considering hydrodynamic conditions which represent storm events at each site, the obtained theoretical results agree fairly well with the field observations. However, in each comparison, the spatial phase shift between the centre of the coarse grains and the topographic low displayed in the observations appears to be not (or only marginally) represented by the theoretical model.

\subsection{Interpretation of the modes}

First, we analyse the results obtained considering the parameter values representative of Wrightsville Beach and Martha's Vineyard (figures 4 and 5, respectively). These results are qualitatively similar, since in both cases $\gamma_{\max , r}$ is positive until a certain wavenumber and then vanishes. Moreover, for both simulations, $\Psi_{\text {ang }}$ is equal to $\pi$.

The obtained results can be understood by taking into account that in both cases only the fine grain size fraction is set into motion. Indeed, for the conditions considered, the shear stress due to the combined action of the current and the waves is smaller than the critical Shields parameter $\theta_{c r, \text { coarse }}^{b}$ for the coarse grains.

Then, if the contributions to the sediment transport of the coarse grain size fraction in the expression of $\mathscr{D}$ (see the Appendix) are set equal to zero, it turns out that $\mathscr{D}$ vanishes. Thus, from (3.35), it follows that $\gamma_{2}$ vanishes for all wavenumbers such that $\gamma_{\max , r}$ is zero after a certain wavenumber (when $\gamma_{1, r}$ becomes negative). Moreover, it is found that $\gamma_{1}$ is equal to $\mathscr{G}$, the real part of which can be written as

$$
\mathscr{G}_{r}=\alpha_{x}\left\{\beta\left(q_{1, h, 2}\right)_{i}-\frac{\beta}{2 L_{a 0}}\left(q_{1, p, 2}\right)_{i}\right\} .
$$

Hence, the growth rate in figures 4 and 5 is given by the linear summation of the imaginary part of the correction to the sediment transport due to perturbations in the bed $\left(q_{1, h, 2}\right)$ and that caused by the spatial changes in the bottom roughness $\left(q_{1, p, 2}\right)$. For simplicity, in the following, the former is denoted by $\gamma_{\text {bed, } r}$ while the latter is represented by $\gamma_{\text {rough }, r}$. 

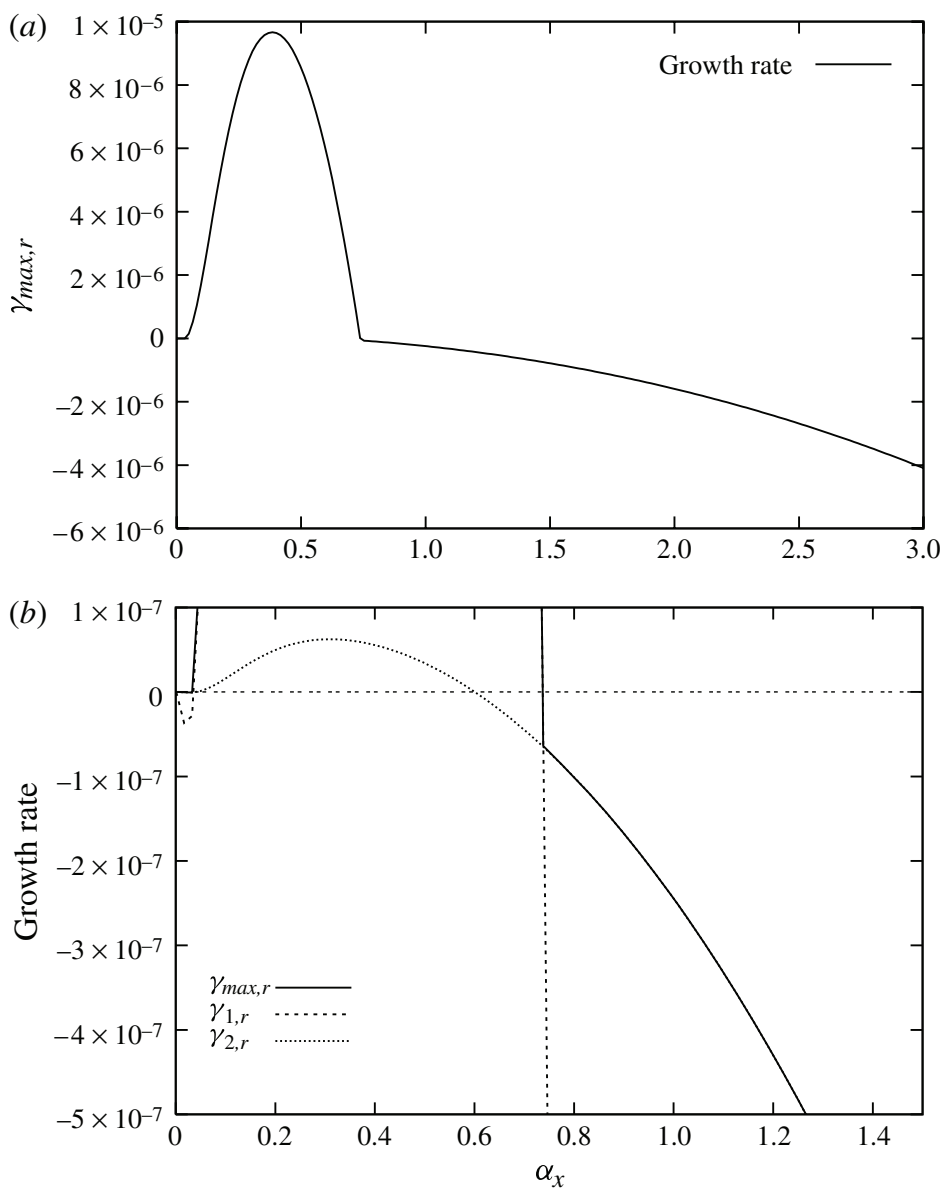

FIGURE 6. (a) Maximum growth rate $\gamma_{\text {max }, r}$ plotted versus the dimensionless wavenumber $\alpha_{x}$ for the conditions off Nauset Inlet, Cape Cod, Massachusetts. (b) The growth rate plotted together with the value of $\gamma_{1, r}$ and $\gamma_{2, r}$. To show the behaviour of $\gamma_{2, r}$, the vertical and horizontal axes have been set smaller, such that the real part of $\gamma_{\max }$ and $\gamma_{2}$ is only partly displayed.

In figure 7 , the contributions of $\gamma_{b e d, r}$ and $\gamma_{\text {rough,r }}$ to the real part of the first eigenvalue are plotted for the input values representative of the sorted bed forms near Wrightsville Beach. The largest contribution to the growth rate is due to the convergence of the sediment transport induced by the perturbations in the bed elevation. Furthermore, figure 7 shows that the perturbations in the bottom roughness, represented by $\gamma_{\text {rough, },}$, tend to destabilise the homogeneously distributed flat bed for bed features characterised by small wavelengths. Figure 7 reveals that, for large values of $\alpha_{x}$, the stabilising character of $\gamma_{\text {bed,r }}$ dominates and $\gamma_{1, r}$ eventually becomes negative.

To analyse the results shown in figure 6 , it is illustrative to compare the results with two extreme theoretical cases. In the first case, the effect of the changes in the bottom composition on the perturbed sediment transport rate is neglected. In the other case, the influence of the perturbation of the bottom elevation on the sediment transport rate is not taken into account. In the former case, from (3.32) it follows that the 


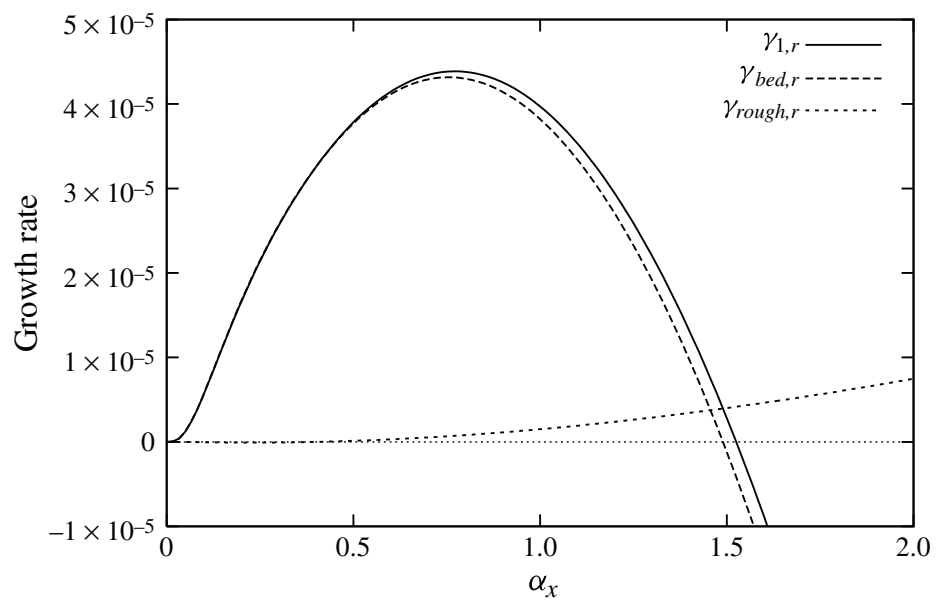

FIGURE 7. The contributions of $\gamma_{\text {bed,r }}$ and $\gamma_{\text {rough }, r}$ to the real part of $\gamma_{1}$ versus $\alpha_{x}$. The parameter values are representative of the inner shelf near Wrightsville Beach.

(in)stability of the morphodynamic system is controlled by the value of the real part $\Gamma_{b e d, r}$, of $\Gamma_{b e d}$ :

$$
\Gamma_{\text {bed }}=-\mathrm{i} \alpha_{x} \beta S_{h} .
$$

If the perturbations of the bed elevation on the sediment transport rate are neglected, the growth rate is determined by the real part of

$$
\Gamma_{\text {rough }}=-\mathrm{i} \alpha_{x} \frac{\beta}{L_{a 0}}\left(q_{1, p, 1}+q_{0,1}-p_{a 0,1} S_{p}\right) .
$$

In figure $8(a)$, the value of $\Gamma_{b e d, r}$ is plotted together with $\gamma_{1, r}$ against the dimensionless wavenumber $\alpha_{x}$, considering the conditions representative of the sorted bed forms described by Aubrey et al. (1982). The growth rate related to the first eigenvalue is found to be mainly determined by the value of $\Gamma_{b e d, r}$. The output of a similar simulation is shown in figure $8(b)$ where the values of $\Gamma_{\text {rough }, r}$ and $\gamma_{2, r}$ are given versus $\alpha_{x}$. Here, the values of $\Gamma_{\text {rough, },}$ and $\gamma_{2, r}$ clearly display different behaviour.

Thus, figure 7 illustrates that the first eigenvalue is almost entirely controlled by the growth of the perturbation of the bottom elevation. In contrast, the second eigenvalue appears to result from a combination of sediment convergence due to perturbations of the bed and the growth of the spatial inhomogeneous bottom composition due to fractional sediment transport related to the perturbations in the bottom roughness. Motivated by these results, we denote the mode corresponding to the first eigenvalue as the 'topography-driven' mode. The second mode will be referred to as the 'roughness-driven' mode. In the following, results of simulations will be shown where $\Gamma_{\text {rough }, r}$ and $\gamma_{2, r}$ agree fairly well such that the term 'roughness-driven mode' for the perturbation associated to the second eigenvalue is more justified.

\subsection{Sensitivity experiments}

The results presented so far have been obtained for hydrodynamic conditions which represent moderate storm conditions at each site. In the following, we investigate what happens if more extreme storm conditions occur, i.e. we consider the effects of increasing the strength of the depth-averaged current or the waveheight. 

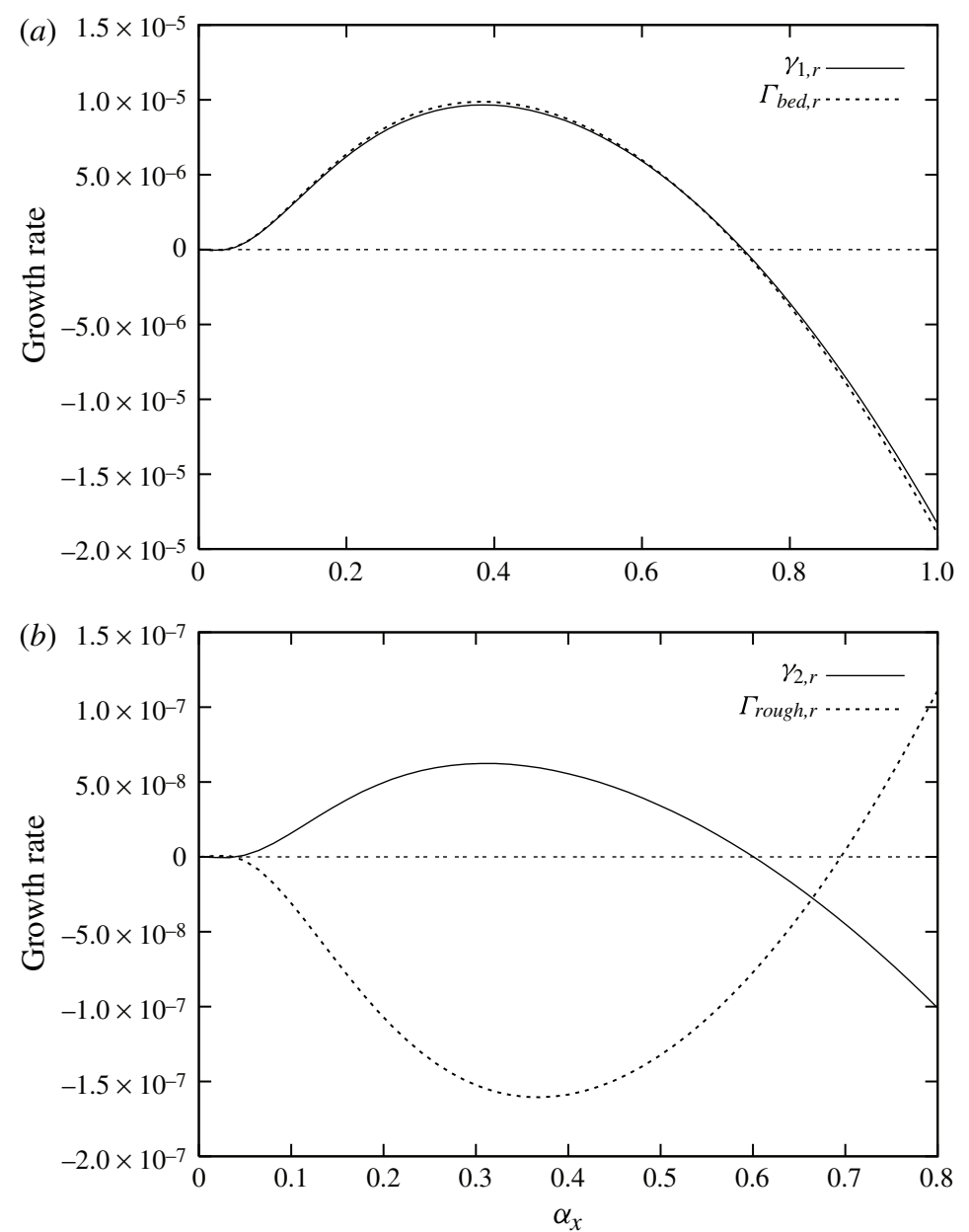

FIGURE 8. (a) Comparison of the real part of the first eigenvalue $\gamma_{1, r}$ with $\Gamma_{b e d, r}$, while in (b) the real part of the second eigenvalue $\gamma_{2, r}$ is compared with $\Gamma_{\text {rough, } r}$. In both figures, the parameter values are representative of the conditions off Nauset Inlet, Cape Cod, Massachusetts.

In figure 9 the growth rate is plotted against $\alpha_{x}$ for different values of $U_{0}^{*}$ for the sorted bed forms off Nauset Inlet. It is found that, when $U_{0}^{*}$ increases, the wavelength of the fastest-growing mode (the topography-driven mode) increases (figure $9 a$ ). Moreover, for larger values of $U_{0}^{*}$, the roughness-driven mode reveals an instability for bed forms with large wavenumbers. As is illustrated in figure $9(b)$, the value of $\gamma_{2, r}$ becomes even larger than the value of $\gamma_{1, r}$ for very large values of $U_{0}^{*}$. In this case, bed features characterised by small wavelengths are generated by the wind-driven current. For example, $\lambda_{\max }^{*}$ is equal to $210 \mathrm{~m}$ for $U_{0}^{*}=0.5 \mathrm{~m} \mathrm{~s}^{-1}$ and it turns out to be $12 \mathrm{~m}$ for $U_{0}^{*}$ equal to $0.6 \mathrm{~m} \mathrm{~s}^{-1}$. Furthermore, the phase shift between the centre of the coarse patches and the bathymetric lows also changes considerably when $U_{0}^{*}$ increases, i.e. when the dominant mode changes. The angle $\Psi_{\text {ang }}$ equals $\pi$ for $U_{0}^{*}=0.5 \mathrm{~m} \mathrm{~s}^{-1}$ and becomes $\pi / 3$ when the depth-averaged mean current is equal to $0.6 \mathrm{~m} \mathrm{~s}^{-1}$. Hence, in the latter simulation, the centre of the coarse region is predicted on the up-current side of the bathymetric low. Note that the migration 

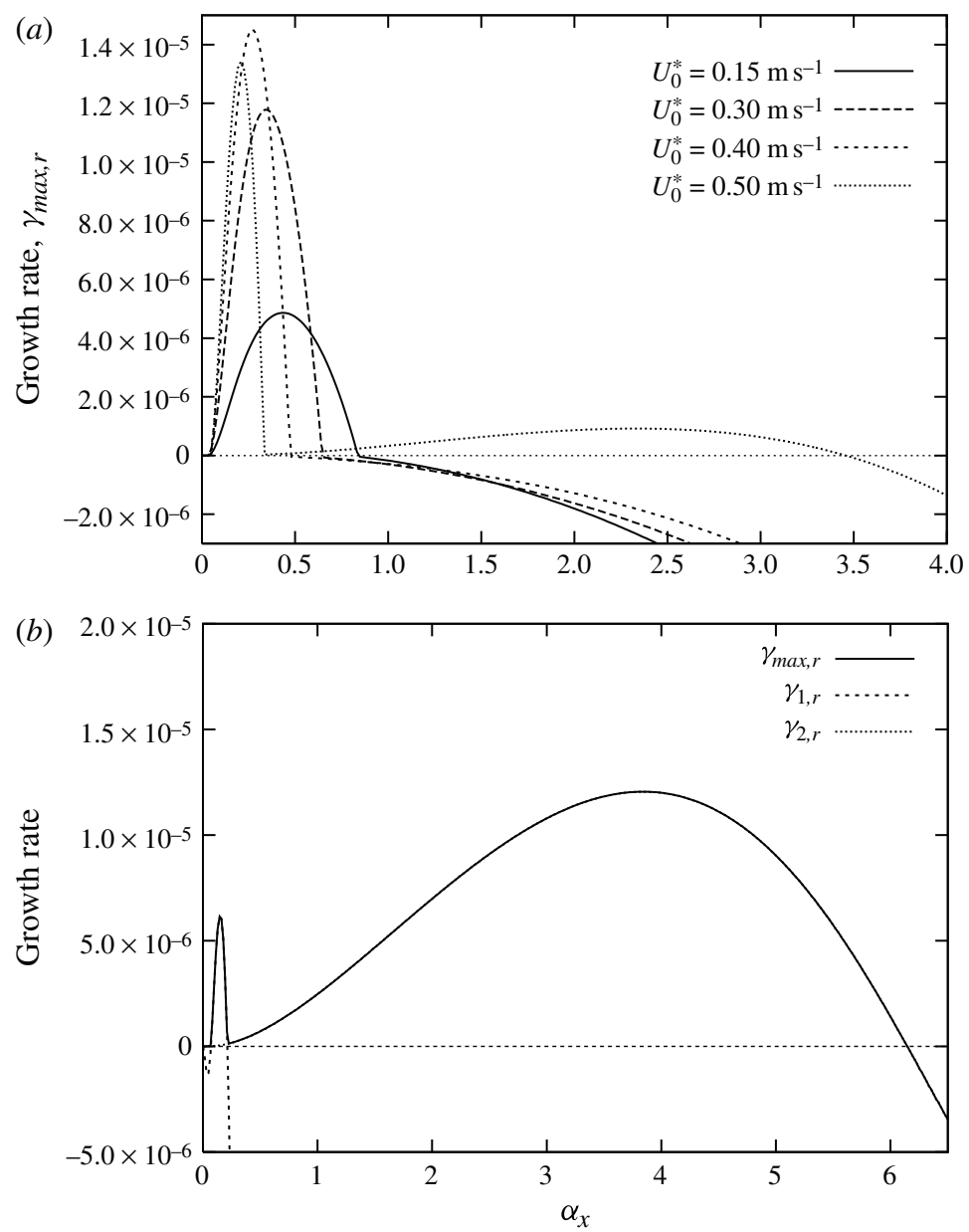

FIGURE 9. (a) Growth rate $\gamma_{\max , r}$ plotted versus the dimensionless wavenumber $\alpha_{x}$ considering the conditions off Nauset Inlet, Cape Cod, Massachusetts and changing the value of $U_{0}^{*}$. (b) The growth rate plotted together with the value of $\gamma_{1, r}$ and $\gamma_{2, r}$ taking the value of $U_{0}^{*}$ equal to $0.6 \mathrm{~m} \mathrm{~s}^{-1}$; the other input values are representative of the conditions off Nauset Inlet.

speed also differs considerably for the two modes. When the topography-driven mode is dominant $\left(U_{0}^{*}=0.5 \mathrm{~m} \mathrm{~s}^{-1}\right) c_{m i g}^{*}$ is equal to $5.7 \mathrm{~m}$ per day, while for $U_{0}^{*}=0.6 \mathrm{~m} \mathrm{~s}^{-1}$ the migration speed of the fastest-growing mode turns out to be approximately $0.75 \mathrm{~m}$ per day. Finally, the amplitude of the ratio between $\tilde{h}$ and $\tilde{p}$ is considered for the topography and roughness-driven modes. For the topography-driven mode, $|\mathscr{R}|$ is of order $10^{-2}$, while for the roughness-driven mode the value of $|\mathscr{R}|$ turns out to be of order $10^{-4}$. Hence, it appears that the bed features related to the roughness-driven mode have an amplitude of the bathymetric undulations, which is much smaller than that of the topography-driven mode.

Finally, considering the conditions off Nauset Inlet with $U_{0}^{*}=0.6 \mathrm{~m} \mathrm{~s}^{-1}$, the value of $\gamma_{1, r}$ is compared with $\Gamma_{\text {bed,r }}$ and the value of $\gamma_{2, r}$ is compared with $\Gamma_{\text {rough }, r}$ in figures $10(a)$ and $10(b)$, respectively. As in figure $8(a)$, the value of $\gamma_{1, r}$ agrees well 

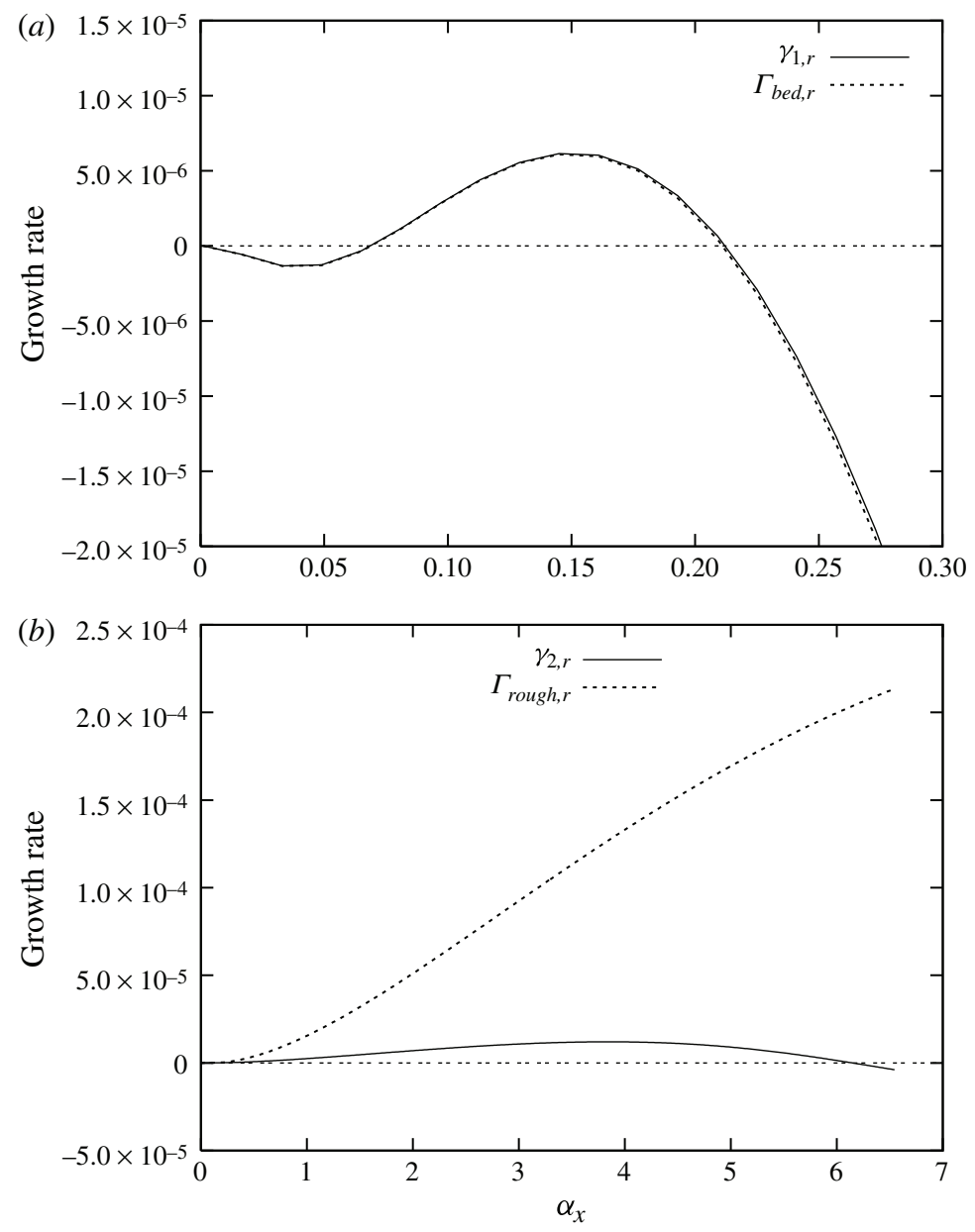

Figure 10. (a) Comparison of the real part of the first eigenvalue $\gamma_{1, r}$ with $\Gamma_{b e d, r}$ and (b) comparison of the real part of the second eigenvalue $\gamma_{2, r}$ with $\Gamma_{\text {rough, }, \text {. The field conditions }}$ off Nauset Inlet with $U_{0}^{*}$ equal to $0.6 \mathrm{~m} \mathrm{~s}^{-1}$ are considered in both figures.

with $\Gamma_{\text {bed,r }}$. For this simulation, the behaviour of $\gamma_{2, r}$ qualitatively resembles $\Gamma_{\text {rough, } r}$ for small and intermediate values of $\alpha_{x}$, but it differs for large wavenumbers.

Similar results are obtained if we consider the conditions at Wrightsville Beach and analyse the influence of different values of the waveheight (see figure 11). Increasing the wave height, first, bed features with larger wavelengths are obtained and the growth rate is dominated by the topography-driven mode. A further increase of $H_{w}^{*}$ leads to the amplification of the roughness-driven mode and to the appearance of bed features with small wavelengths. As in the previous simulation, the migration speed of the fastest-growing mode decreases significantly when the roughness-driven mode becomes dominant. In particular, $c_{\text {mig }}^{*}$ turns out to be $\sim 1 \mathrm{~m}$ in three months, considering $H_{w}^{*}=3 \mathrm{~m}$, and it is found to be of order $1 \mathrm{~m}$ per day when the topography-driven mode is dominant.

In contrast to the results presented in figure $9(b)$, for the simulations with $H_{w}^{*}=3 \mathrm{~m}$, the value of $\Psi_{\text {ang }}$ turns out to be approximately $1.2 \pi$, yielding a shift of the centre of 


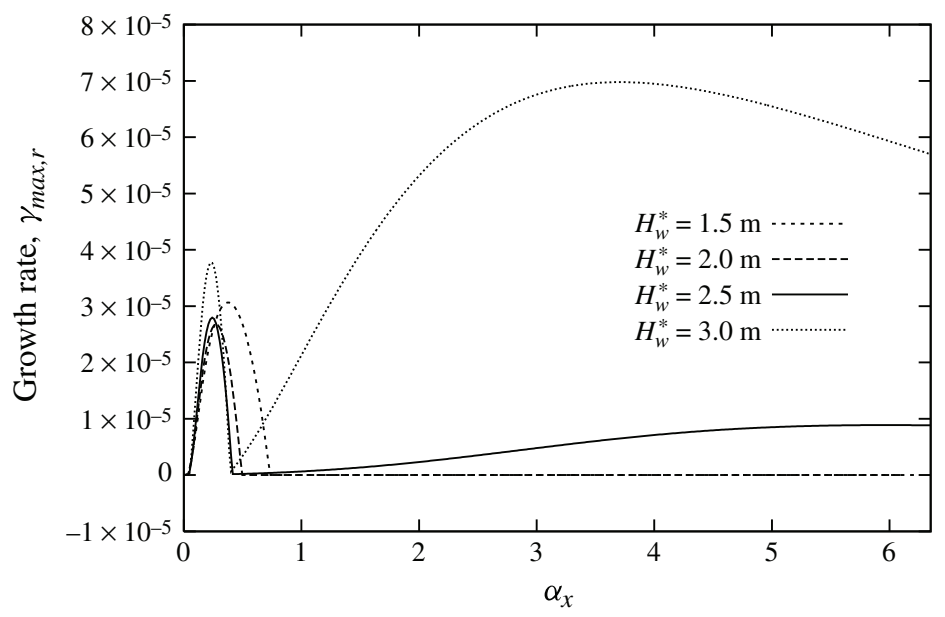

FIGURE 11. The value of $\gamma_{\max , r}$ against $\alpha_{x}$ considering input values representative of the inner shelf near Wrightsville Beach. Different values of the waveheight are considered.

the coarse grains down-current of the bathymetric low. Moreover, for these simulations, the amplitude of the ratio between $\tilde{h}$ and $\tilde{p}$ is of order $10^{-2}$.

\section{Discussion}

The results previously presented show that the inherent feedback between winddriven currents, waves, and a bottom consisting of a heterogeneous sediment, on a shelf with a constant depth, can lead to the amplification of two distinct modes. The first mode is almost entirely controlled by the correction of the sediment transport due to changes of the bed elevation. The second mode emerges as the result of the interaction between the convergence of sediment induced by the perturbations of the bed elevation and by the inhomogeneous sediment transport related to changes in the bottom composition. The former (topography-driven) mode leads to the amplification of bed forms with relatively large wavelengths (order of a hundred metres) in which the centre of the coarse patch occurs in the troughs of the bottom waviness. The ratio between the amplitude of the perturbation of the bed elevation and that of the bottom composition is $O\left(10^{-2}\right)$ and the migration speed ranges between 1 and $10 \mathrm{~m}$ per day. The topography-driven mode appears to be dominant when the wave climate is moderate and intermediate values of the steady depth-averaged current velocity are considered. Moreover, this mode also emerges when the hydrodynamic conditions are such that only the fine sediment is set into motion.

Increasing the waveheight and/or considering large values of $U_{0}^{*}$, bed features, related to the roughness-driven mode, with smaller wavelengths (order of tens of metres) tend to amplify and eventually dominate over the topography-driven mode. This finding is similar to the results described by Seminara et al. (1996) for fluvial environments where, for increasing values of the Froude number, a bedsheet mode appears, which eventually becomes dominant over the dune mode. Taking into account different values of the input parameters, the roughness-driven mode can display both 
an up-current and a down-current shift of the centre of the coarse grain size region with respect to the bathymetric low. Furthermore, the value of $|\mathscr{R}|$ ranges from values much smaller than those resulting from the topography-driven mode up to values which are comparable. Finally, the migration speed of the roughness-driven mode ranges between 0.1 and $1 \mathrm{~m}$ per day.

Since the results are obtained within the framework of a linear stability analysis, conclusions founded on the model findings regarding the profile and characteristics of the bed features in the field should be treated with care, especially taking into account that previous numerical models suggest that the resulting wavelength is considerably affected by nonlinear interactions (Murray \& Thieler 2004; Coco et al. 2007a). Nevertheless, the study provides information on the free response of the morphodynamic system, which can be an indicator of the observed features (Dodd et al. 2003). In this respect, the analysis suggests that the broad range of characteristics observed in the field, even within one study area, could be the result of a competition between the two modes, in which slight changes of the hydrodynamic, bathymetric or sedimentologic conditions might lead to a shift in the dominant mode and therefore also to changes of the characteristics of the observed bed features. For instance, this interpretation could explain the observations of sorted bed forms at Martha's Vineyard, reported by Goff et al. (2005), where the bed features in the western part of the study area are characterised by an amplitude which is of the order of $10 \mathrm{~cm}$, and display a different asymmetry from the bed forms on the eastern side, which are characterised by amplitudes up to $3 \mathrm{~m}$.

Although the analysis is only valid for small, infinitesimal perturbations and therefore cannot capture the nonlinear competition between the modes, to illustrate the above idea it is interesting to consider the input values representative of the conditions off Nauset Inlet, together with $U_{0}^{*}=0.6 \mathrm{~m} \mathrm{~s}^{-1}$ and different water depths (figure 12). In this case, the amplification rate of bed features with small wavelengths decreases with respect to the topography-driven mode and eventually vanishes entirely when $h_{0}^{*}$ is increased. For $h_{0}^{*}=18 \mathrm{~m}$, the fastest-growing mode turns out to have a wavelength equal to $\sim 85 \mathrm{~m}$, which is 5 times larger than the wavelength of the most amplified mode when $h_{0}^{*}$ is equal to $7 \mathrm{~m}$. When water depths larger than $8 \mathrm{~m}$ are considered, the value of $|\mathscr{R}|$ is much larger (order 10) than the value obtained for $h_{0}^{*}=7 \mathrm{~m}$. Hence, the shift from a dominant roughness-driven mode to a dominant topography-driven mode could explain the observation that, offshore of the $8 \mathrm{~m}$ contour, the sorted bed forms have much larger wavelengths and are related to bathymetric undulations with larger amplitudes than the bed features observed in shallower waters.

In spite of the results obtained, some aspects of the model results are subject to further study. First, the roughness-driven mode is found to be dominant only for very high values of $U_{0}^{*}$ and $H_{w}^{*}$, which probably rarely occur. Moreover, the growth rate related to the topography-driven mode is found to attain the largest maximum for conditions representative of storm events at each site, while observations of sorted bed forms in the field reveal that the bed features exhibit characteristics of the roughnessdriven mode (e.g. asymmetry) over a broad range of conditions.

Another issue to discuss is that figure 12 (for $h_{0}^{*}=10 \mathrm{~m}, 14 \mathrm{~m}$ ) reveals that there are conditions for which the roughness-driven mode does not attain a maximum and the value of $\gamma_{2, r}$ keeps on increasing for smaller and smaller wavelengths. In these cases, the analysis does not provide a fastest-growing wavelength and thus fails. To quantify the conditions in which the second eigenvalue does not attain a maximum, in figure 13 a contour plot of the value of $\lambda_{\max }^{*}$ is shown considering different values of $U_{0}^{*}$ and $H_{w}^{*}$ and the sedimentologic and bathymetric input values representative 


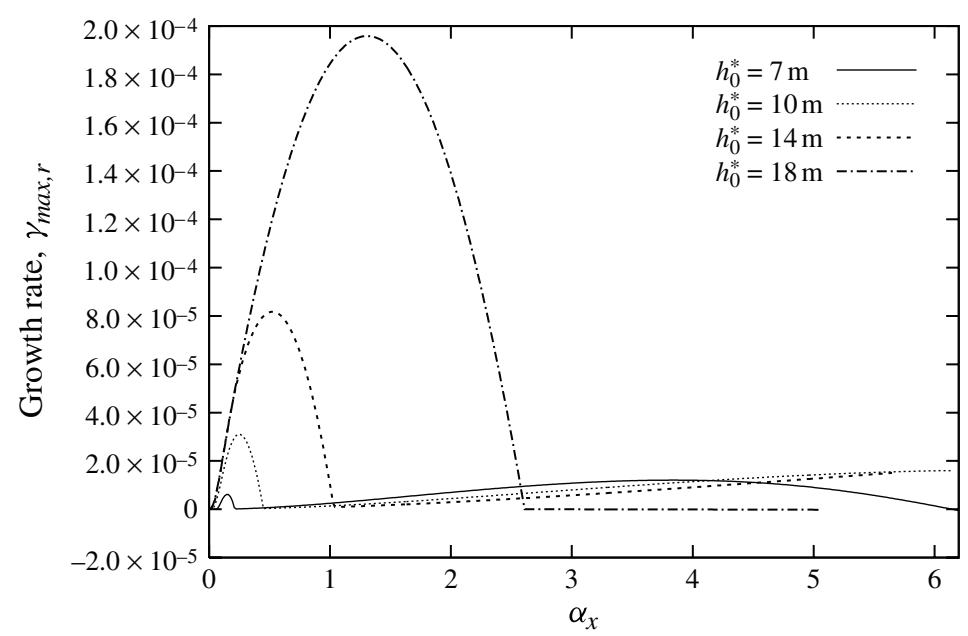

FIGURE 12. The maximum growth rate plotted versus $\alpha_{x}$, considering different values of $h_{0}^{*}$. The input values representative of Nauset Inlet with $U_{0}^{*}=0.6 \mathrm{~m} \mathrm{~s}^{-1}$ are taken into account.

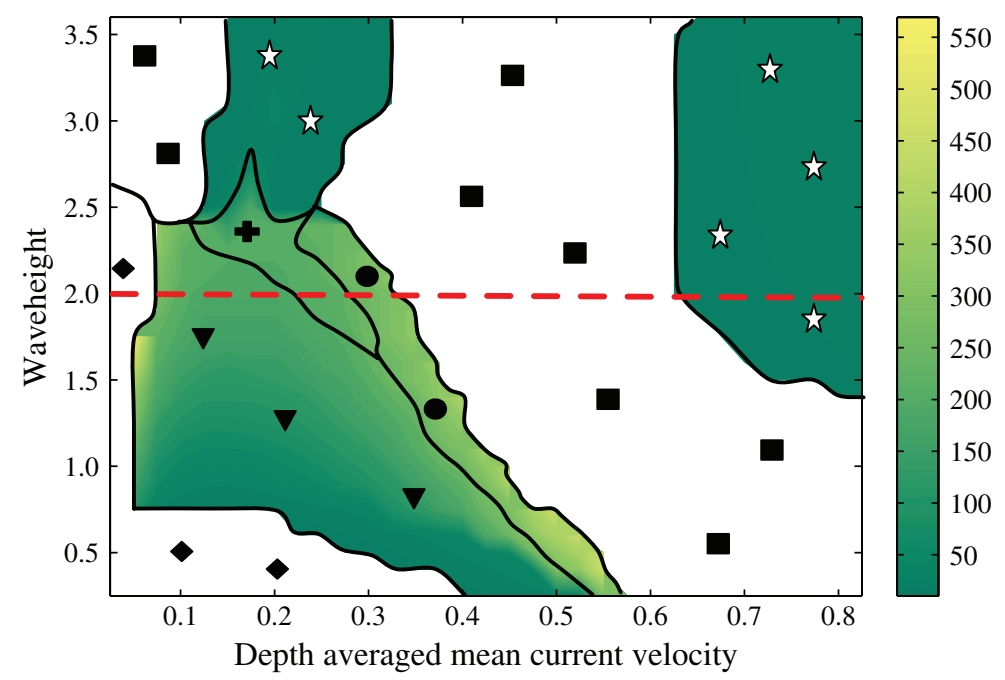

FIgURE 13. Contour plot of the value of $\lambda_{\max }^{*}(\mathrm{~m})$ in dependence of $U_{0}^{*}$ and $H_{w}^{*}$ on the horizontal and vertical axes, respectively. Bathymetric and sedimentologic input values representative of Wrightsville Beach are considered. The white region with $\square$ symbols denotes conditions where the roughness mode is dominant but no maximum is attained. The region with $\star$ indicates that the fastest-growing mode is related to the real part of the roughness-driven mode. No growth of bed features is predicted for the white area with $\diamond$ and only one grain size fraction is set into motion in the region indicated by $\nabla$. The region with + symbols indicates conditions in which the topography-driven mode is dominant and the second eigenvalue attains a maximum, while in the area with $\bullet$ the roughness-driven mode does not attain a maximum but the topography-driven mode is dominant for all wavenumbers.

of Wrightsville Beach. A similar contour plot, in which values representative of Nauset inlet are taken into account, is given in Van Oyen (2010). Several regions are indicated in the contour plot, which correspond to different qualitative situations. 

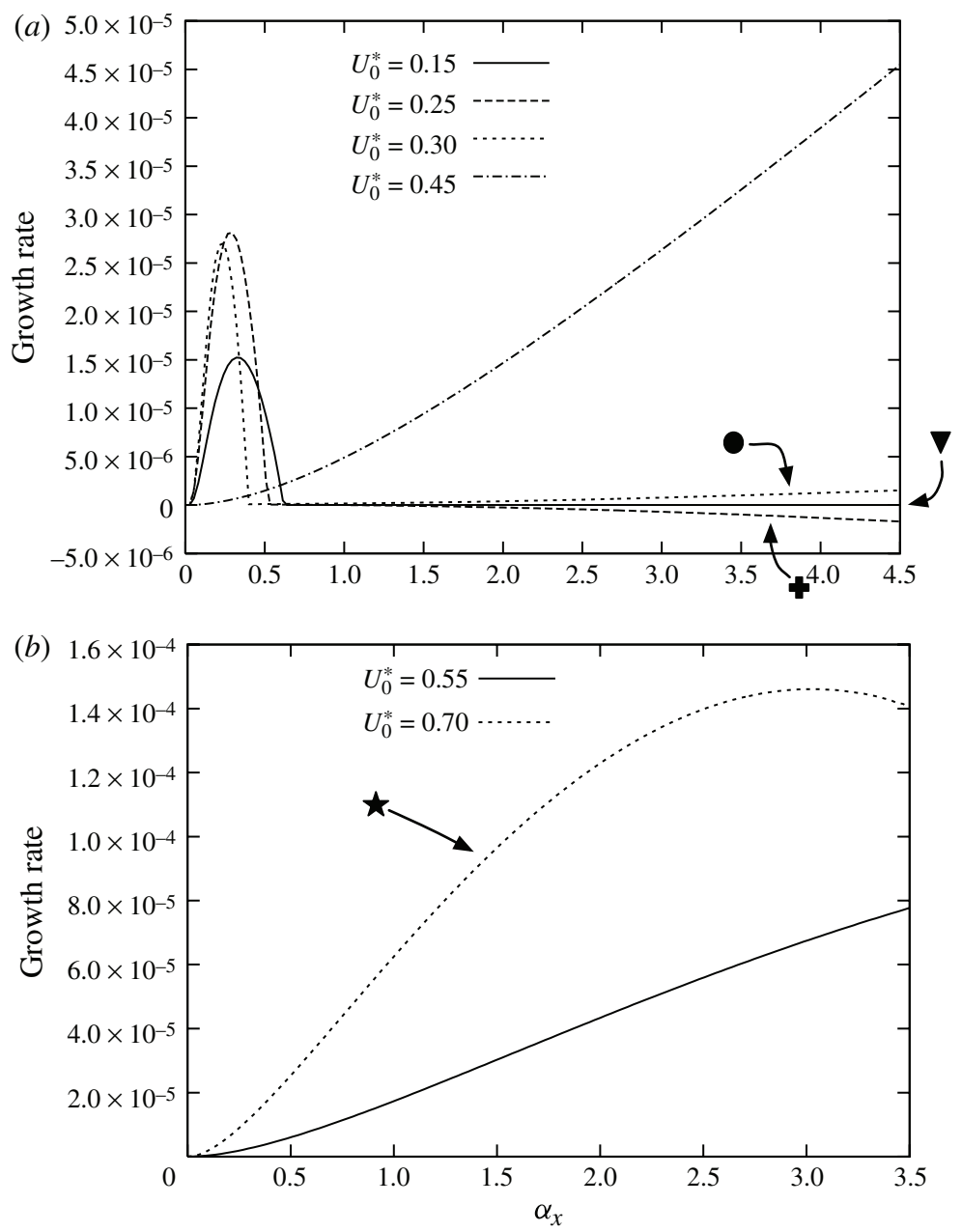

FIGURE 14. The value of $\gamma_{\max , r}$ plotted versus $\alpha_{x}$ considering the input values representative for Wrightsville Beach with $H_{w}^{*}=2.0 \mathrm{~m}$ and different strengths of the steady current.

In the white region with diamonds $(\diamond)$, the sediment is not set into motion and no bed features are amplified. The white region with squares $(\square)$ denotes conditions in which the roughness-driven mode is dominant over the topography-driven mode, but the growth rate does not attain a maximum in the investigated range of wavenumbers (wavelengths). In these conditions, no most amplified mode can be chosen on the basis of the present analysis. In the coloured region, the value of $\lambda_{\max }^{*}$ is represented in the colour bar next to the contour plot. The region with stars $(\star)$ indicates the conditions which lead to the roughness-driven mode. In the area with + symbols the topographydriven mode is dominant and both eigenvalues attain a maximum. The region indicated by triangles $(\nabla)$ represents conditions where there is only one grain size fraction set into motion and the analysis predicts the appearance of the topography-driven mode. Finally, the region with dots $(\bullet)$ represents the situation in which the roughness-driven mode does not attain a maximum, but the maximum of the amplification rate due to the first eigenvalue remains larger than the real part of $\gamma_{2, r}$ for all the wavenumbers 

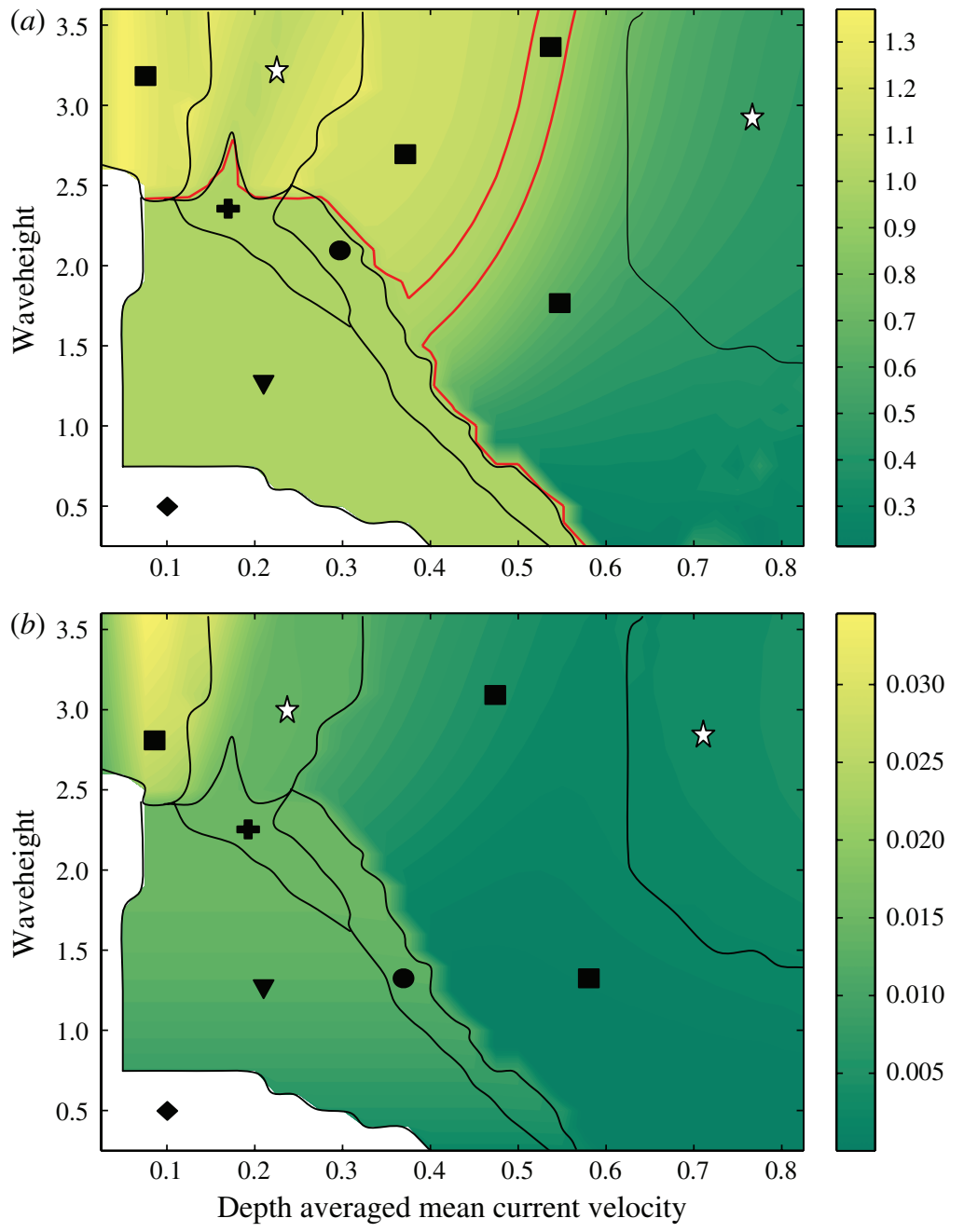

FIGURE 15. (a) Contour plot of the phase shift between the centre of the coarse patch and the trough of the bathymetric undulation. Values of $\Psi_{\text {ang }}$ smaller (larger) than one correspond to a phase shift up-current (down-current). The red lines denote transition over values of $1.05 \pi$ and $0.95 \pi$. (b) Contour plot of the amplitude of $\mathscr{R}$. In both figures, the input values are representative for Wrightsville Beach. An explanation for the notation of the different regions is provided in the text and in the caption of figure 14.

for which a convergent solution is found. In this case, the value of $\lambda_{\max }^{*}$ shown in the contour plot is related to the wavenumber for which the topography-driven mode attains a maximum.

To illustrate more clearly the behaviour of the growth rate for each region, in figure 14 , the value of $\gamma_{\max , r}$ is plotted versus $\alpha_{x}$ considering the input values representative of Wrightsville Beach together with $H_{w}^{*}=2.0 \mathrm{~m}$ and different values of $U_{0}^{*}$; i.e. we follow the broken line shown in figure 13 .

Finally, in figure 15 the changes in the value of $\Psi_{\text {ang }}$ and in the amplitude of $\mathscr{R}$ for different hydrodynamic conditions are illustrated considering the input values representative of Wrightsville Beach. The value of $\Psi_{\text {ang }}$ is illustrated in figure 15(a) 
by plotting the amount with which $\pi$ should be multiplied to obtain the value of $\Psi_{\text {ang. }}$. Hence, values smaller (larger) than one correspond to a phase shift up-current (down-current). For the regions where the roughness-driven mode is dominant and it does not attain a maximum, the values of $\Psi_{\text {ang }}$ and $|\mathscr{R}|$ are evaluated for $\alpha_{x}$ equal to 4 . The choice to represent these results for $\alpha_{x}$ equal to 4 is, of course, arbitrary. However, when the roughness-driven mode is dominant, the phase shift between the bathymetric low and the coarse patch, and the amplitude of $\mathscr{R}$ is found only to differ slightly in the range of wavenumbers under consideration (not shown). We refer to Van Oyen (2010) for similar contour plots considering values of the input parameters representative for Nauset Inlet.

We also performed additional simulations, in which we excluded hiding effects in the sediment transport formulation. The results (not shown) indicate that ignoring hiding leads to smaller growth rates of the topographically induced modes, whilst the growth rate of the roughness-induced mode (when present) increases.

\section{Conclusions}

In conclusion, the model appears to be able to explain the appearance of sorted bed forms in the coastal region. Indeed, the analysis reveals that the positive coupling between waves, a steady along-shore current, and an erodible heterogeneous bed leads to the amplification of two modes. One mode (topography-driven mode) is almost entirely related to the convergence of the sediment transport rate due to the perturbation of the bed elevation. A second mode (roughness-driven mode) is found to be generated by a combination of the correction of the sediment transport due to the bottom perturbation and that related to the perturbation of the bottom composition. In particular, the model suggests that the broad range of characteristics displayed by sorted bed forms in the field could be the result of a competition between these two modes. On the other hand, for a significant range of hydrodynamic conditions, the model does not provide a fastest-growing mode. Moreover, the roughness-driven mode is dominant only for high values of the waveheight $H_{w}^{*}$ and for strong alongshore currents $U_{0}^{*}$. Therefore, it appears that the present model cannot be considered conclusive, although it provides basic insight into the physical mechanisms controlling the growth and migration of sorted bed forms and explains many aspects of features observed in the field.

This research has been supported by the Ministero dell'Università e della Ricerca. Initial stage funding was also provided by the University of Genova. The first author wishes to acknowledge the European Union for a grant in the framework of the FLUBIO project.

\section{Appendix}

The expressions of $S_{p}$ and $S_{h}$ appearing in (3.30) are given by

$$
\begin{gathered}
S_{p}=p_{a 0,1}\left(q_{b 1, p, 1}+q_{s 1, p, 1}\right)+p_{a 0,2}\left(q_{b 1, p, 2}+q_{s 1, p, 2}\right)+q_{b 0,1}+q_{s 0,1}-q_{b 0,2}-q_{s 0,2} \\
S_{h}=p_{a 0,1}\left(q_{b 1, h, 1}+q_{s 1, h, 1}+q_{s l 1, h, 1}^{b}\right)+p_{a 0,2}\left(q_{b 1, h, 2}+q_{s 1, h, 2}+q_{s l 1, h, 2}^{b}\right) .
\end{gathered}
$$

The components of the $2 \times 2$ matrix appearing in (3.34) read

$$
\mathscr{L}_{p 1}=-\mathrm{i} \alpha_{x} \frac{\beta}{L_{a 0}}\left(q_{1, p, 1}+q_{0,1}-p_{a 0,1} S_{p}\right), \quad \mathscr{L}_{p 2}=-\mathrm{i} \alpha_{x} \beta S_{p},
$$




$$
\mathscr{L}_{h 1}=-\mathrm{i} \alpha_{x} \frac{\beta}{L_{a 0}}\left(q_{1, h, 1}-p_{a 0,1} S_{h}\right) \quad \text { and } \quad \mathscr{L}_{h 2}=-\mathrm{i} \alpha_{x} \beta S_{h} .
$$

The expressions of $\mathscr{G}$ and $\mathscr{D}$ appearing in (3.35) can be written as

$$
\mathscr{G}=\mathscr{L}_{h 2}+\mathscr{L}_{p 1}, \quad \mathscr{D}=\mathscr{L}_{p 1} \mathscr{L}_{h 2}-\mathscr{L}_{p 2} \mathscr{L}_{h 1}
$$

\section{REFERENCES}

AshidA, K. \& Michiue, M. 1972 Study on hydraulic resistance and bedload transport rate in alluvial streams. Trans. Japan Soc. Civil Engrs 206, 59-69.

Aubrey, D. G., Twichell, D. C. \& Pfirman, S. L. 1982 Holocene sedimentation in the shallow nearshore zone off Nauset inlet, Cape Cod, Massachusetts. Mar. Geol. 47, 243-259.

Bellec, V. K., Be, R., Rise, L., Slagstad, D., Longva, O. \& Dolan, M. F. J. 2010 Rippled scour depressions on continental shelf bank slopes off Nordland and Troms, northern Norway. Cont. Shelf Res. 1056-1069.

Brownlie, W. R. 1981 Prediction of flow depth and sediment discharge in open channels. Tech Rep. W.M. Keck Lab. of Hydraulics and Water Resources, California Institute of Technology, Pasadena, California.

Cacchione, D. A., Grant, W. D. \& TAte, G. B. 1984 Rippled scour depressions on the inner continental shelf off central California. J. Sedim. Petrol. 54, 1280-1291.

Christoffersen, J. V. \& Jonsson, I. G. 1985 Bed friction and dissipation in a combined current and wave motion. Ocean Engng 12, 387-423.

Coco, G., Murray, A. B. \& Green, M. O. $2007 a$ Sorted bed forms as self-organized patterns. Part 1. Model development. J. Geophys. Res. 112, F03015.

Coco, G., Murray, A. B., Green, M. O., Thieler, E. R. \& Hume, T. M. 2007b Sorted bed forms as self-organized patterns. Part 2. Complex forcing scenarios. J. Geophys. Res. 112, F03016.

Colombini, M. 2004 Revisiting the linear theory of sand dune formation. J. Fluid Mech. 502, $1-16$.

Colombini, M. \& PARKer, G. 1995 Longitudinal streaks. J. Fluid Mech. 304, 161-183.

Colombini, M. \& Stocchino, A. 2005 Wind effect in turbulence parametrization. Adv. Water Resour. 28, 939-949.

DEAN, R. D. 1974 Aero report 74-11. Tech Rep. Imperial College, London.

Diesing, M., Kubicki, A., Winter, C. \& Schwarzer, K. 2006 Decadal scale stability of sorted bedforms, German Bight, southeastern North Sea. Cont. Shelf. Res. 26, 902-916.

Dodd, N. P., Blondeaux, P., Calvete, D., Falqués, A., De Swart, H. E., Hulscher, S. J. M. H., ROZYNSKI, G. \& VitTORI, G. 2003 Understanding coastal morphodynamics using stability methods. J. Coast. Res. 19, 849-865.

FERRINI, V. L. \& FLOOD, R. D. 2005 A comparison of rippled scour depressions identified with multibeam sonar: evidence of sediment transport in inner shelf environments. Cont. Shelf. Res. 25, 1979-1995.

Fredsøe, J. \& DeigaARD, R. 1992 Mechanics of Coastal Sediment Transport, Advanced Series on Ocean Engineering, vol. 3. World Scientific.

Goff, J. A., Mayer, L. A., Traykovski, P., Buynevich, I., Wilkens, R., Raymond, R., Glang, G., Evans, R. L., Olson, H. \& Jenkins, C. 2005 Detailed investigation of sorted bedforms, or 'rippled scour depressions,' within the Martha's Vineyard coastal observatory, Massachusetts. Cont. Shelf. Res. 25, 461-484.

Gutierrez, B. T., Voulgaris, G. \& Thieler, E. R. 2005 Exploring the persistence of sorted bedforms on the inner-shelf of Wrightsville beach, North Carolina. Cont. Shelf. Res. 25 $65-90$.

Hirano, M. 1971 On river bed degradation with armouring. Trans. Japan Soc. Civil Engrs 3 (2), 194-195.

Kleinhans, M. G. \& VAN RiJn, L. C. 2002 Stochastic prediction of sediment transport in sand-gravel bed rivers. J. Hydraul. Engng 128, 412-425. 
Kovacs, A. \& PARKer, G. 1994 A new vectorial bedload formulation and its application to the time evolution of straight river channels. J. Fluid Mech. 267, 153-183.

Lanzoni, S. \& Tubino, M. 1999 Grain sorting and bar instability. J. Fluid Mech. 393, 149-174.

Murray, A. B., Coco, G., Green, M. O., Hume, T. \& Thieler, E. R. 2005 Different approaches to modeling inner shelf sorted bedforms. In 4th IAHR Symposium on River, Coastal and Estuarine Morphodynamics (ed. G. Parker \& M. Garcia), pp. 1009-1015.

Murray, A. B. \& Thieler, E. R. 2004 A new hypothesis and exploratory model for the formation of large-scale inner-shelf sediment sorting and 'rippled scour depressions'. Cont. Shelf. Res. 24, 295-315.

PARKER, G. 1978 Self-formed straight rivers with equilibrium banks and mobile bed. Part 1. The sand-silt river. J. Fluid Mech. 89, 109-125.

VAN RiJn, L. C. 1984 Sediment transport. Part 2. Suspended load transport. J. Hydraul. Engng 110, $1613-1641$.

VAN RIJN, L. C. 1991 Sediment transport in combined currents and waves. In Sand Transport in Rivers, Estuaries and the Sea (ed. Soulsby \& Bettess). Euromech 262.

VAN RIJn, L. C. 1993 Principles of Sediment Transport in Rivers, Estuaries and Coastal Seas. Aqua.

Schwab, W. C., Thieler, E. R., Allen, J. R., Foster, D. S., Swift, B. A. \& Denny, J. F. 2000 Influence of inner-continental shelf geologic framework on the evolution and behavior of the barrier-island system between Fire Island Inlet and Shinnecock Inlet, Long Island, New York. J. Coast. Res. 16, 408-422.

Seminara, G., Colombini, M. \& PARKer, G. 1996 Nearly pure sorting waves and formation of bedload sheets. J. Fluid Mech. 312, 253-278.

Soulsby, R. L. 1997 Dynamics of Marine Sands. Thomas Telford.

Soulsby, R. L. \& Whitehouse, J. S. 2005 Prediction of ripple properties in shelf seas. Tech Rep. 150. Wallingford Ltd.

De Swart, H. E., Walgreen, M., Calvete, D. \& Vis-Star, N. C. 2008 Nonlinear modelling of shoreface-connected ridges: impact of grain sorting and interventions. Coast. Engng 55, 642-656.

Thieler, E. R., Plikey, O. H., Cleary, W. J. \& Schwab, W. C. 2001 Modern sedimentation on the shoreface and inner continental shelf at Wrightsville beach, North Carolina, USA. J. Sedim. Res. 71, 958-970.

Thieler, E. R., Schwab, W. C. \& Cacchione, D. A. 1998 Wave and current measurements in a cross-shore scour depression on the shoreface off Wrightsville beach, North Carolina. Eos Trans. AGU 79 (1) supplement, OS61.

VAN DORN, W. C. 1953 Wind stress on an artificial pond. J. Mar. Res. 12, 249-276.

VAN OYEN, T. 2010 Sediment sorting in coastal seas. PhD thesis, University of Genoa.

VAn Oyen, T. \& Blondeaux, P. 2009 Grain sorting effects on the formation of tidal sand waves. J. Fluid Mech. 629, 311-342.

VAN OYEn, T., DE SWART, H. E. \& BlondeauX, P. 2010 Bottom topography and roughness variations as triggering mechanisms to the formation of sorted bedforms. Geophys. Res. Lett. 37, L18401.

Vis-Star, N. C., De Swart, H. E. \& CAlvete, D. 2009 Effect of wave-bedform feedbacks on the formation of, and grain sorting over shoreface-connected sand ridges. Ocean Dyn 59, 731-749.

Walgreen, M., de Swart, H. E. \& Calvete, D. 2003 Effect of grain size sorting on the formation of shoreface-connected sand ridges. J. Geophys. Res. 108, C3. 